Article

\title{
Feature Representation and Data Augmentation for Human Activity Classification Based on Wearable IMU Sensor Data Using a Deep LSTM Neural Network
}

\author{
Odongo Steven Eyobu ${ }^{1,2}$ (1) and Dong Seog Han ${ }^{1, *(1)}$ \\ 1 School of Electronics Engineering, Kyungpook National University, 80 Daehak-ro, Buk-gu, Daegu 41566, \\ Korea; sodongo@knu.ac.kr \\ 2 School of Computing \& Informatics Technology, Makerere University, Plot 56, Pool Road, P.O. Box 7062, \\ Kampala, Uganda \\ * Correspondence: dshan@knu.ac.kr; Tel.: +82-53-950-6609
}

Received: 16 July 2018; Accepted: 27 August 2018; Published: 31 August 2018

\begin{abstract}
Wearable inertial measurement unit (IMU) sensors are powerful enablers for acquisition of motion data. Specifically, in human activity recognition (HAR), IMU sensor data collected from human motion are categorically combined to formulate datasets that can be used for learning human activities. However, successful learning of human activities from motion data involves the design and use of proper feature representations of IMU sensor data and suitable classifiers. Furthermore, the scarcity of labelled data is an impeding factor in the process of understanding the performance capabilities of data-driven learning models. To tackle these challenges, two primary contributions are in this article: first; by using raw IMU sensor data, a spectrogram-based feature extraction approach is proposed. Second, an ensemble of data augmentations in feature space is proposed to take care of the data scarcity problem. Performance tests were conducted on a deep long term short term memory (LSTM) neural network architecture to explore the influence of feature representations and the augmentations on activity recognition accuracy. The proposed feature extraction approach combined with the data augmentation ensemble produces state-of-the-art accuracy results in HAR. A performance evaluation of each augmentation approach is performed to show the influence on classification accuracy. Finally, in addition to using our own dataset, the proposed data augmentation technique is evaluated against the University of California, Irvine (UCI) public online HAR dataset and yields state-of-the-art accuracy results at various learning rates.
\end{abstract}

Keywords: human activity recognition; data augmentation; feature representation; deep learning; long short term memory; inertial measurement unit sensor

\section{Introduction}

Current technological advancements in microelectronics have ushered in the design and manufacture of wireless miniature devices with key capabilities including the ability to house wearable inertial measurement unit (IMU) sensors, and wireless transmission capability. At the centre of this technological development is the desire for such miniature devices to be used in applications such as ambient assisted living (AAL) [1], physiological medical diagnostics [2], localization and navigation [3-5], mobile and wireless context-driven decision support systems [6], and security monitoring. The data that IMU sensors are capable of generating ranges from gyroscope, accelerometer, and magnetometer to global positioning system (GPS) information, depending on 
the product limitations. It is from this possibility of acquiring these IMU data that data-driven and knowledge-based learning models are relevant for data discrimination.

Deep learning models are data-driven learning models. Studies $[7,8]$ have shown that deep learning models are able to learn and discriminate among human activities ranging from sitting, walking, climbing upstairs, walking down-stairs and falling, among others. However, studies [8] report that certain activities which produce relatively stationary data are challenging to discriminate amongst. Examples of such activities include sitting, standing and lying down (facing left, facing right, facing up and facing down) [9]. The challenge in discriminating relatively stationary data of different activities arises because of similarity of their feature representations. For example; when such data are considered as signals, transformations to the frequency domain may show relatively similar spectral information. It is for such a reason that proper feature extraction mechanisms are very important for classification purposes.

Statistical parameters and convolutions are the popular conventions for feature representation of data for learning purposes. Statistical parameters are popular for time-series data whereas convolutions are popular in image processing studies. However, various studies including $[7,10]$ preferred to utilise a combination of statistical features and frequency domain features for data representation. Preferred combinations are always a choice geared towards improving the quality of the feature vector. To this end, the key point in a feature representation approach is in its quality that should represent the intrinsic characteristic of each class of data. Conventional approaches used for dimension reduction of data include principal component analysis (PCA) [11,12], and convolution approaches using defined kernels [7].

In this paper, a feature extraction approach is proposed. The features are extracted from a spectrogram of the 3-dimensional (3D) raw acceleration and gyroscope data collected in a defined period of seconds. A set of least and largest values from the spectrogram is selected to represent the data abstraction referred to as the feature vector used to training the deep learning model. The least and greatest value set selection approach is a kind of dimension reduction approach applied to the feature vector without compromising much on the originality of the data in this paper.

A feature representation for a given class can only be considered exhaustive qualitywise if all or a huge percentage of raw data variants of the same class are represented. Apart from just having few samples of training data as a classification problem, it is also reported both in practice and literature [13] that sensors exhibit a displacement problem during the process of data collection and testing even when the sensor is put at the same human body position during data collection for training and testing. The displacement problem is one of the causes of false classifications. The obvious solution to this problem is collecting a lot of training data while accounting for sensor displacement. This is a very tedious process, hence a challenge in human activity recognition (HAR) studies. One powerful solution for this problem is to perform data augmentation.

Data augmentation provides an opportunity to create deformations of training samples without changing the semantics of the raw data. Such deformations are added to the training dataset with the objective of representing unseen raw data. Data deformation is a popular practice in image processing where transformations such as image rotation and image scaling pose similar significant semantic meaning of the original image. However; for wearable sensor data, it is very challenging to generate suiting deformations that maintain the semantics of the data label. This rigidity is because the data variations are an intensity factor of motion. Therefore, depending on the kinds of activities under study, a deformation may alter the semantic meaning of the label. Nevertheless, data augmentation has been successfully applied in wearable sensor data in studies such as [13,14] showing positive state-of-the-art results, and sound recognition [15]. It should be noted that data augmentation for wearable sensor data has not been exhaustively and systematically investigated unlike augmentations for image, sound and speech recognition. In this article, a data augmentation procedure for wireless IMU sensor data is proposed. The major data augmentation machinery used is the down-sampling approach based on local averaging. In addition to local averaging, data shuffling is done to cause data 
variations in the feature vector sets to enable further local averaging of the same data and to reduce on overfitting.

Apart from the feature representation as a challenge in classification problems, the number of classes to be dealt with in an experiment is also a challenge as classification results tend to be skewed to some class or classes due to data imbalance [16-18]. This phenomenon is common in binary classification $[19,20]$ where only two classes are involved. However, the same challenge presents itself in the classification of three classes as well. In this paper, experiments are conducted to recognize only three activities with the objective of determining the influence of the proposed augmentation approach on the data imbalance and overfitting problem.

In this paper, the problem of classifying human activities using data from wearable IMU sensors is handled using a deep recurrent neural network (RNN) with long short term memory (LSTM). The LSTM neural network is tweaked with a greedy-wise hyper-parameter adjustment for the learning rates and feature vector size in order to understand the network performance.

The original contributions of this paper can be summarized as follows: (1) a proposed feature extraction algorithm whose abstraction is based on defined least and largest spectral values, (2) an ensemble of feature space augmentation methods applicable to wearable IMU sensor data and suitable for human activity classification and (3) an experimental analysis of both the proposed feature extraction and an ensemble of augmentations to understand the influence of each method on classification accuracy. The rest of this article is organized as follows: Section 2 discusses the related works in HAR and data augmentation. Section 3 presents the proposed feature extraction algorithm and augmentation method. Section 4 presents the experimental setup. Section 5 presents the results and discussions. Finally, Section 6 concludes this article.

\section{Related Works}

HAR using wearable IMU sensors exhibits possible diverse application areas. For this reason, HAR studies are very attractive nowadays. Already, various domain specific studies seeking to utilize HAR based on wearable sensors have been conducted and more are still emerging. Therefore, in this section, selected HAR studies are categorically reviewed with the interest of showing existing feature set selection and classification methods for time series data in the following order: (1) HAR for AAL (elderly care, human behaviour understanding), and (2) HAR for healthy living (fitness for preventive health care). Given the method applied in this article, a subsection with keen interest on feature representation and data augmentation studies in HAR that use deep learning is presented to end the current state-of-the-art and acts as an introduction to the proposed method in this article.

\subsection{HAR for $A A L$}

The vision of AAL is that persons should be able to live life independently regardless of their physical weakness and human disabilities. Assistive technologies are the key drivers behind this vision. Fall detection in elderly people [21-23] is seen to be the most popular and attractive application in AAL studies. Tremor caused by diseases like Parkinson's have been quantitatively assessed in studies like [24] to aid quick diagnosis and remedies. The possibility of understanding human intention using motion data has also been studied in [25] as a build-up to achieve AAL.

In [21], both the Kinect sensor and a wearable motion-sensing device are used to detect falls. The combination is aimed at minimizing the number of false alarms. In their system, a small number of false alarms are achieved owing to visual validation of the fall alert generated based on motion data only. A total of 612 images were used for training from the University of Rzeszow (UR) fall detection dataset (URFD). For classification of the fall, the support vector machine (SVM) was used. The feature sets used for classification purposes and analysis included: the colour depth maps only, and colour depth + acceleration features. However, it should be noted that since the Kinect sensor is a vision based system, it is naturally limited by privacy controls. Cameras cannot be placed in some locations 
such as bathrooms, so when an elderly person falls while taking a bath in the absence of a camera, only the wearable sensor can be useful.

Mao et al. [22] proposed a fall detection method based on the acceleration and Euler angle data extracted from a wearable micro electro-mechanical system (MEMS) sensor to represent the orientation of the users' body using Kalman solutions. Through experiments where the sensors were placed on the subjects' shoulder, waist and foot, a threshold of acceleration was identified for accurate fall detection. In other words, the threshold of acceleration acted as the key feature for classification. However, despite of the accuracies achieved in the threshold approach, using adaptive thresholding would be more robust for fall detection. This is solely because different human beings have different structures naturally especially as they grow old. The natural body orientation of an elderly person may be somehow curved. Such cases would raise false alarms. Pierleoni et al. [23] proposed a fall detection algorithm which was implemented in a wearable device. Their system utilises a fusion of triaxial accelerometer, gyroscope and magnetometer data from an IMU sensor. Based on the root mean square (RMS), yaw, pitch and roll, the orientation of the subject can be specified to determine whether it's a fall or not. In [24], a quantitative assessment of Parkinson's tremor is conducted using least-square-estimation models. Tremor quantification was based on a time-frequency signal features got from IMU data.

Apart from using only inertial sensors for motion analysis, recent studies [26] have considered the fusion of both inertial sensors and camera vision sensors to improve on the human activity recognition accuracy. Vision-based activity recognition would require that feature extraction approaches for image data must be carefully considered to enable accurate activity detection. Various feature extraction approaches in image processing include using the spatio-temporal interest point (STIP) [27] detector, motion-energy images (MEI) and motion history images (MHI) [28].

Human gait modeling studies [29] have also come up with descriptive motion models which can be used to aid the recognition of persons and activities. Some descriptive gait models are based on imagery, animation and inertial sensor data to determine the human posture, motion, stride length, and stance time [30].

\subsection{HAR for Healthy Living}

Apart from selectivity in what to eat, a healthy body needs exercise to remain fit. Fitness-oriented applications focus on this need by being able to automatically identify human activities, log them and do statistical analyses for body fitness assessments [31]. In order to achieve the latter, wearable devices such as smart watches or smartphones are equipped with inertial sensors to generate data and user analysis software applications for feedback purposes [32]. Sports-related activities include swimming, jogging, walking, jumping, push-ups, running, playing football, roller skating among others. In $[7,8]$, activities such as walking, standing, sitting, laying, walking upstairs, cycling, jogging were recognized based on a dep convolutional neural network $(\mathrm{CNN})$ learning methodology. Basing on convolution theory, the features used in both studies are a result of applying defined filters to the original data set in [8] and to the spectrogram in [7].

In [33], a data analysis tool called SwimMaster was developed with the capability of identifying the swimming style, swimming stroke counter, body balance and rotation. Inertial sensors were mounted on the swimmers' upper arm, the lower back and the right wrist for data collection purposes. Evaluation of the study parameters was based on an analysis of the yaw, roll and pitch values as discriminating features.

In summary, time series classification approaches as observed in the review above can be split in two major categories: (1) distance-based (SVM, $k$ nearest neighbors $(k-\mathrm{NN})$, least square estimations (LSE), Euclidean distances) and (2) feature-based methods (Fourier coefficients, spectrograms, logic regression, means, variance) being the fastest in training and most popular nowadays. The proposed classification approach in this article is feature-based with spectral information being extracted for classification. 


\subsection{Feature Representation Studies}

Features are generally abstractions of data. The main purpose of feature extraction is to find abstractions from a data segment that can accurately represents the original data. In other words, the transformation of large input data into a reduced representation set of features, which can also be referred as feature vector, is called feature extraction [34]. Feature vectors include discriminating information between various activities or classes of data. It is the feature vectors that are used as inputs to classification algorithms. The Table 1 shows various feature representation approaches from various studies and their applications.

Table 1. Some of the most widely used features as discussed in [34].

\begin{tabular}{lll}
\hline \multicolumn{1}{c}{ Type } & \multicolumn{1}{c}{ Features } & \multicolumn{1}{c}{ Some Applications } \\
\hline Time-domain & $\begin{array}{l}\text { Mean [35], variance, standard deviation [36,37], } \\
\text { root mean square [38,39], zero or mean crossing } \\
\text { rate [40], derivative, peak counts [41,42] }\end{array}$ & $\begin{array}{l}\text { Human activity recognition } \\
{[37,38], \text { speech recognition [39], }} \\
\text { eye movement analysis [42] }\end{array}$ \\
\hline Frequency-domain & $\begin{array}{l}\text { Discrete fast Fourier transforms coefficient, } \\
\text { spectral energy [7,43] }\end{array}$ & Human activity recognition [7] \\
\hline Time frequency domain & Wavelet coefficients [44] & Blink detection [42] \\
\hline
\end{tabular}

In Ravi et al. [7] the features are derived by first generating a spectrogram of the raw data and then performing a convolution process to generate the data abstraction which represents the feature vector. In contrast to Ravi et al. [7], the proposed feature representation approach in this article also uses spectrogram data and generates the data abstraction set of data based on defined least and greatest values of the same spectral data.

\subsection{Data Augmentation Studies}

Currently, there are indeed limited studies reported in literature that use or even address data augmentation as a mechanism for improving time series data classification accuracy for wearable sensors. Some of the existing methods are mentioned in this subsection. Guennec et al. [45] proposed window slicing and dynamic time warping (DTW). Cui et al. [46] as well proposed window slicing for data augmentation which is developed as follows: For a time series $T=\left\{t_{1}, \ldots, t_{n}\right\}$, a slice is a snippet of the original time series, defined a $S_{i: j}=\left\{t_{i}, t_{i+1}, \ldots, t_{j}\right\}, 1 \leq i \leq j \leq n$. Suppose a time series $T$ is of length $n$, and the length of the slice is $s$, the slicing operation will generate a set of $n-s+1$ sliced time series:

$$
\operatorname{Slicing}(T, s)=\left\{S_{1: s,} S_{2: s+1}, \ldots, S_{n-s+1: n}\right\}
$$

where all the time series in Slicing $(T, s)$ have the same label as their original time series $T$ does.

In [14], a sizeable number of data augmentation methods for time series data are mentioned and implemented. These include:

(1) Rotations: To cater for multiple sensor placement scenarios which represent the same label, controlled data rotation may offer generalization ability of such unseen data. An example of such a scenario is when a sensor is placed upside down compared to its normal position during collection of training data.

(2) Permutation: This is a method to perturb the temporal location of with-in window events. To perturb the location of data in a single window, the data are first sliced into $\mathrm{N}$ same length segments. The segments are then randomly permutated to create a new window.

(3) Time-warping: Is another approach used to perturb the temporal location of data. This is done by smoothly distorting the time intervals between samples. This is like time scale modification (TSM) whereby the window can be compressed by reducing the time interval of samples or extended by increasing on the time interval between samples. 
(4) Scaling: This approach involves changing the magnitude of the data in a window by applying a random scalar.

(5) Magnitude-warping: Involves changing the magnitude of each sample by convolving the data window with a smooth curve varying around one.

(6) Jittering: Involves including additive sensor noise.

It can be seen that most popular augmentations in literature are done in data space. However, recently, feature space augmentation approaches are being proposed. Some feature space augmentation studies are in studies like [47-49]. The synthetic minority over-sampling technique (SMOTE) by [49] is the most popular of these. In this article, we propose augmentation by local averaging which is further improved by data shuffling. Our augmentation approach is done in feature space and logically explained in the next section together with the feature extraction approach describing our proposed method.

\section{Proposed Approach}

This study is based on the paradigm illustrated by Figure 1. The contributions in this study are in the feature extraction block and data augmentation block. In the feature extraction block, the short Fourier transform (STFT) is used as a tool to generate a spectrogram from which the spectral information is extracted. The STFT is performed on IMU sensor raw data generated in a time window period of $3 \mathrm{~s}$ and later on its subsequent time overlaps. The spectral information is shaped as a vector which is reduced in length by sorting and extracting a set of defined least and greatest values hence forming the data abstraction. The manipulation to reduce on the size of the spectral information is motivated by the fact that large input feature vectors cause a long training time for a deep learning system compared to shorter feature vectors. The manipulated spectral feature vectors are finally used for training a deep learning system. The detailed description of the feature extraction process is explained in Section 3.1.

In the data augmentation block, two techniques are used. These are the local averaging as a down-sampling technique and shuffling. Local averages of the spectral feature dataset based on a defined criteria is calculated and then appended at the tail end of the feature set. Shuffling of feature vectors is done to create variation in the data in the case where further downsampling is desired. The detailed description of the augmentation procedure is explained in Section 3.2.

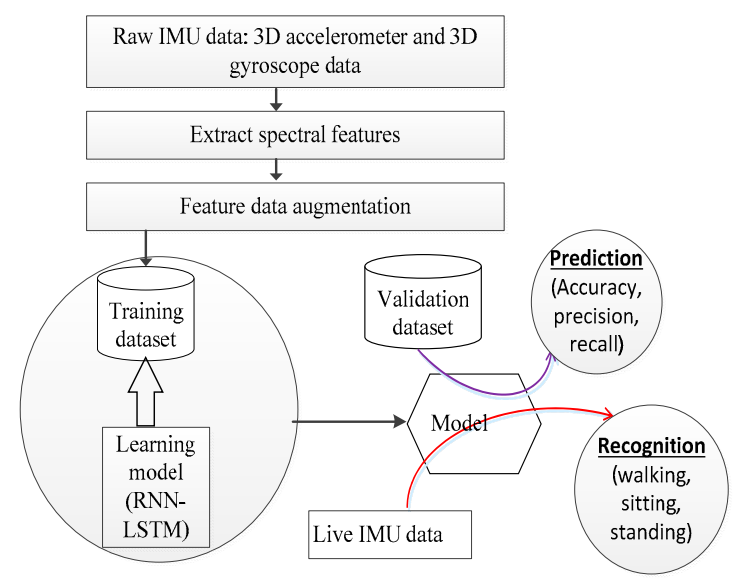

Figure 1. Human activity recognition system workflow.

\subsection{Feature Extraction Method}

Consider 3D accelerometer and gyroscope data extracted from an IMU sensor based on the experimental architecture in Figure 2. All triaxial accelerometer and gyroscope information from the 
IMU sensor are received at a server for learning and classification purposes. Figure $3 a, b$ show the raw triaxial data extracts from sitting and walking scenarios in a period of $10 \mathrm{~s}$. Figure $4 \mathrm{a}$ is an extraction of a traw riaxial walking signal from Figure 3 and showing its spectogram for a period of $3 \mathrm{~s}$ hence representing the interval for the activity recognition.

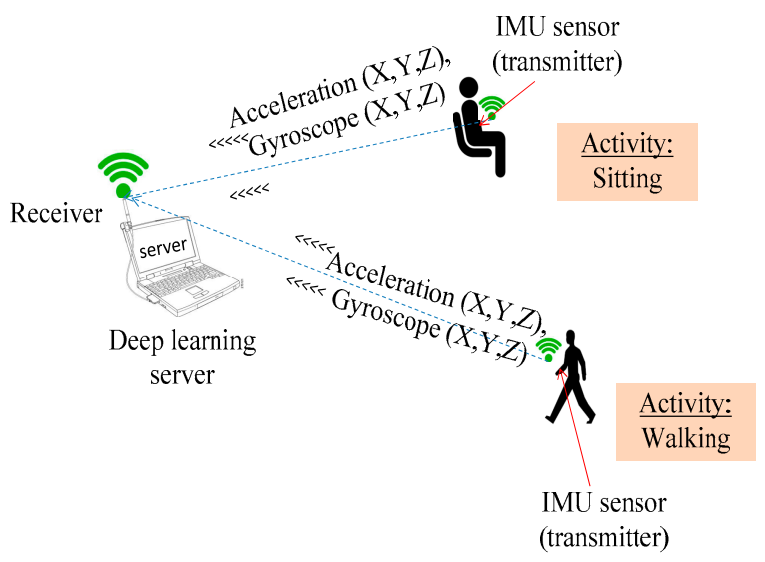

Figure 2. Data collection architecture.

Figure $4 \mathrm{~b}$ shows the workflow for the proposed feature extraction whose input are the spectrogram information generated from Figure $4 \mathrm{a}$. It should be noted that subsequent windows from each of the Figure $4 \mathrm{a}$ data are generated by overlapping the previous window by $50 \%$ in order to generate other spectrograms for continuous feature extraction. Therefore, the STFT for each window extract can be generated by:

$$
\operatorname{STFT}\{k[n]\}(m, \omega)=\sum_{n=-\infty}^{\infty} k[n] W[n-m] e^{-j \omega n}
$$

The spectrogram is then described as $|\operatorname{STFT}(n, \omega)|^{2}$ and $k[n]$ can be any one of the 3D (X or $Y$ or $Z$ ) raw signals at time $n, m$ is the time shift applied to the window used in the transformation expressed as $W[n]$. For this study, the Hanning window was used and the number of data points used in each FFT block is 512 with a sampling frequency of $50 \mathrm{~Hz}$. The Hanning window function is popularly used because a signal reconstruction close to the original signal is achievable.

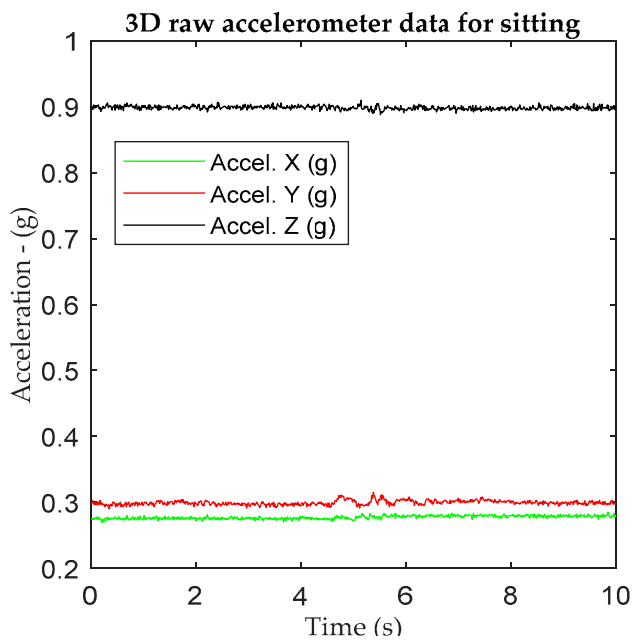

(a)

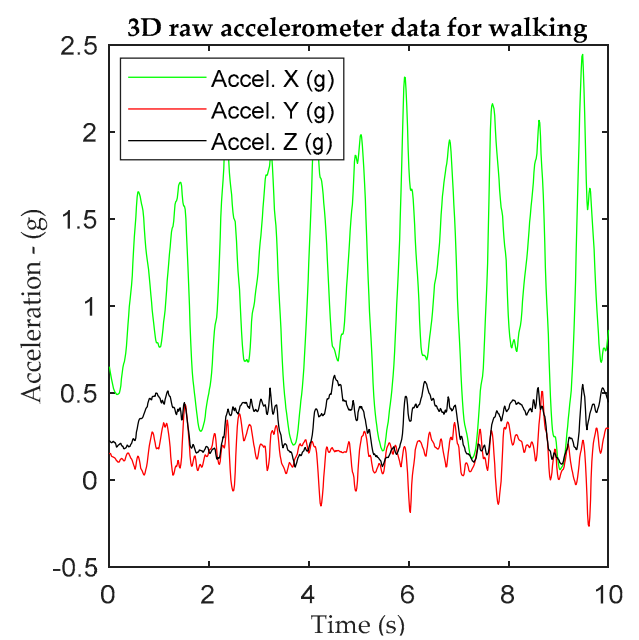

(b)

Figure 3. An example of 3D raw data for (a) sitting and (b) walking based on 1IMU sensor tied on the left-hand wrist. 

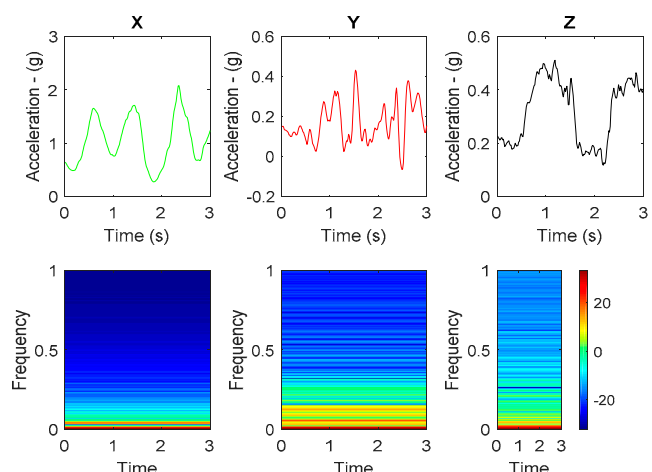

(a)

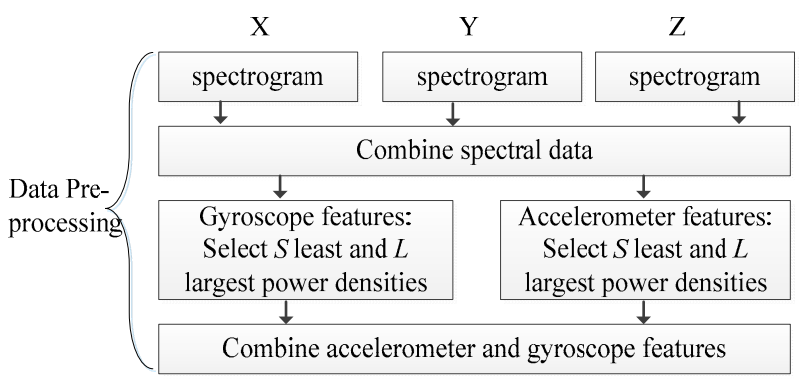

(b)

Figure 4. (a) An example of time domain data and their spectrogram representing walking data; (b) Proposed feature extraction algorithm.

At each window, when the spectrogram for each dimension is generated, the power densities in each spectrogram representation are all combined and sorted in an ascending order. A set of power densities comprising of the least and another set comprising of the largest power densities are considered as features of the data set. In this paper, the number of the least power denstities are referred to as $S$ and the number of largest power densities are referred to as $L$. The generated $S$ and $L$ data for both the gyroscope and the accelerometer are combined ready for input to the learning module. Therefore, for this study, when $S$ and $L$ are each equal to 25 for both the accelerometer and gyroscope spectral data, then we generate a 100-feature vector dataset. In the same vain, when $S$ and $L$ are each equal to 50 for both the accelerometer and gyroscope spectral data, then we generate a 200 -feature vector dataset. The 100-feature vector and 200-feature vector datasets are in this article referred to either as the initial feature set or our dataset in Section 5.

We are motivated to use the least and largest of the spectral information on the basis that spectrogram information in its raw form can be used to generate features using approaches like convolution successfully as seen in various literature such as [7]. In essence, by convolution, it is possible to extract the salient features in the spectrogram to represent the data. It is in the same vain that we can use the same raw spectral data without transforming it but carefully set the boundaries or limiting factors for selecting those to be used as features. The least and largest values of a specified size were used. Our approach to use the least and largest spectral value set for spectral data abstraction is motivated by the fact that the convolution process presents a larger latency compared to the selection process of least and largest spectral information. The latency factor is important when considering efficient real time processing.

\subsection{Augmentation in Feature Space}

Augmentation is done after generation of the spectral features dataset based on the proposed feature extraction approach. The augmentation workflow is illustrated in Figure 5 and in Tables 2-7. As seen in the Figure 6, there exist two main techniques applied in the study to generate augmentation 
data. These are down-sampling by local averaging and shuffling. To walk-through the augmentation process, Figures 6-11 representations based on Figure 5 are described next.

The extracted spectral features dataset is represented by Figure 6. Figure 7 shows the column-wise local average generating process. Figure 8 contains both data in Figure 6 and locally averaged data which appears at the tail end. Figure 9 shows the data in Figure 8 that has been shuffled row-wise to create variation in local averaging in the next local averaging process seen in Figure 10. Figure 11 shows an augmented dataset resulting from the original spectral features set, local averaged data, shuffling process and another local averaging process. The next paragraph puts into context the data representation in Figures 6-11 with respect to the augmentation procedure.

If we consider an initial feature set as a matrix of data points, with each row representing a sample containing the least acceleration PSD data (SA $i$ ), largest acceleration PSD data (LeA $i$ ), least gyroscope PSD data (SGi), largest gyroscope PSD data (LGi) where $i$ is the sample number, then data can be down sampled by averaging the columns to formulate the augmentation data (MSA1, MLeA1, MSG1, MLG1) ... (MSAL, MLeAL, MSGL, MLGL) seen in Figure 8. In this study, we first down sample the four items continuously for all columns in Figure 7 for formulating the new samples to append to the feature set. The new feature set seen in Figure 8 is then shuffled, and down sampled again by averaging only two column items continuously for all the columns as seen in Figure 10. The augmentation data with feature vectors $\left(\mathrm{MC}_{11}, \mathrm{MC}_{12}, \mathrm{MD}_{11}, \mathrm{MD}_{12}\right) \ldots\left(\mathrm{MC}_{\mathrm{K} 1}, \mathrm{MC}_{\mathrm{K} 2}, \mathrm{MD}_{\mathrm{K} 1}, \mathrm{MD}_{\mathrm{K} 2}\right)$ are then appended to the shuffled data represented in Figure 8 to formulate the augmented data in Figure 11.

It should be noted that the augmentation process followed in this article is done for each class independently. The following notations and descriptions are used in this analysis: OR-Original spectral features, LA1-1st local averaging, SH—shuffling, LA2—2nd local averaging, ST—Standing, SI-Sitting, and WA-Walking.

Shuffling is done in machine learning systems to reduce on the variance of data especially when dealing with mini batch processing hence making the model to remain general and overfit less [50]. For the study experiment, row-wise random shuffling was used. The random shuffles were performed for each class exclusively.

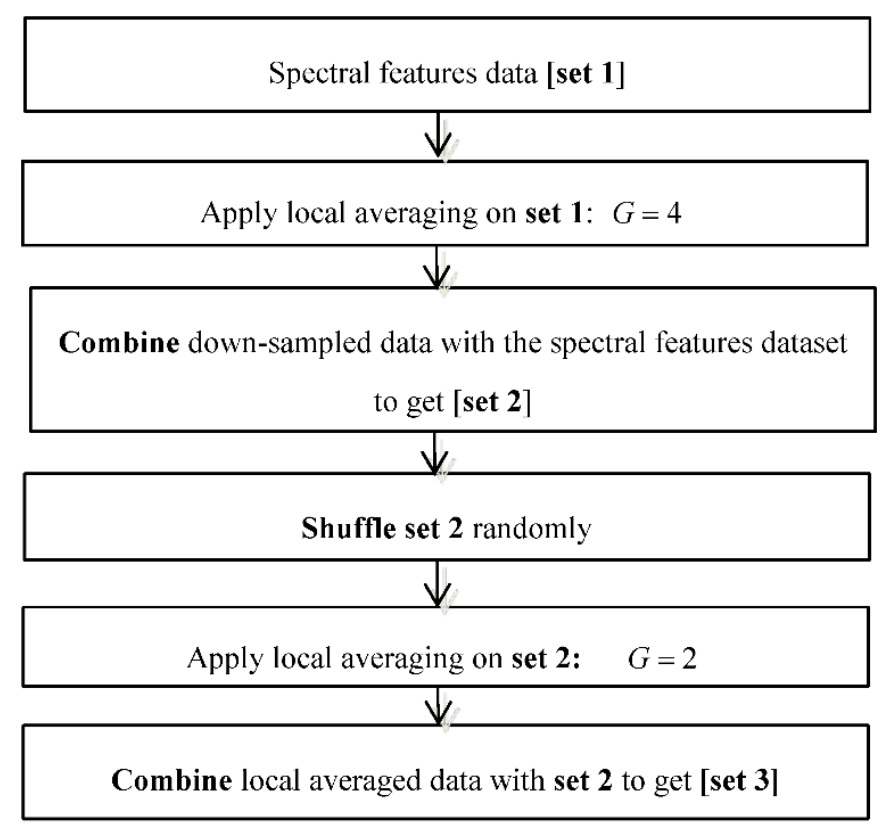

Figure 5. Data augmentation workflow. 


\begin{tabular}{|c|c|c|c|}
\hline $\mathrm{SA}_{1}$ & $\mathrm{LeA}_{1}$ & $\mathrm{SG}_{1}$ & $\mathrm{LG}_{1}$ \\
\hline $\mathrm{SA}_{2}$ & $\mathrm{LeA}_{2}$ & $\mathrm{SG}_{2}$ & $\mathrm{LG}_{2}$ \\
\hline $\mathrm{SA}_{3}$ & $\mathrm{LeA}_{3}$ & $\mathrm{SG}_{3}$ & $\mathrm{LG}_{3}$ \\
\hline $\mathrm{SA}_{4}$ & $\mathrm{LeA}_{4}$ & $\mathrm{SG}_{4}$ & $\mathrm{LG}_{4}$ \\
\hline $\mathrm{SA}_{5}$ & $\mathrm{LeA}_{5}$ & $\mathrm{SG}_{5}$ & $\mathrm{LG}_{5}$ \\
\hline $\mathrm{SA}_{6}$ & $\mathrm{LeA}_{6}$ & $\mathrm{SG}_{6}$ & $\mathrm{LG}_{6}$ \\
\hline $\mathrm{SA} 7$ & $\mathrm{LeA} 7$ & $\mathrm{SG}_{7}$ & $\mathrm{LG}_{7}$ \\
\hline . & . & . & . \\
\hline . & . & . & . \\
\hline . & . & . & . \\
\hline $\mathrm{SA}_{n-1}$ & $\operatorname{LeA}_{n-1}$ & $\mathrm{SG}_{n-1}$ & $\mathrm{LG}_{n-1}$ \\
\hline $\mathrm{SA}_{n}$ & $\mathrm{LeA}_{n}$ & $\mathrm{SG}_{n}$ & $\mathrm{LG}_{n}$ \\
\hline \multicolumn{4}{|c|}{ 小 } \\
\hline \multirow{2}{*}{\multicolumn{2}{|c|}{ Accelerometer's }} & \multirow{2}{*}{\multicolumn{2}{|c|}{$\begin{array}{c}\text { Gyroscopes' } \\
\text { spectral data } \\
\text { Size } S \text { and size } L\end{array}$}} \\
\hline Size $S$ & & & \\
\hline
\end{tabular}

Figure 6. Set 1: OR dataset.

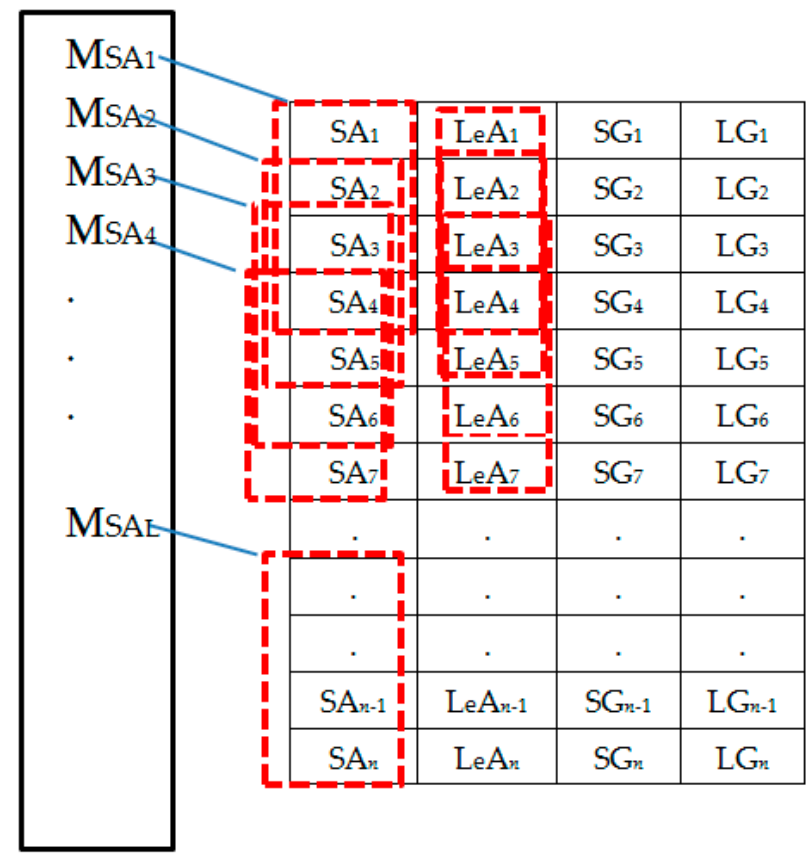

$G=4:$ Local averages

Figure 7. Set 1: Generating local averages. 


\begin{tabular}{|c|c|c|c|}
\hline $\mathrm{SA}_{1}$ & $\mathrm{LeA}_{1}$ & $\mathrm{SG}_{1}$ & $\mathrm{LG}_{1}$ \\
\hline $\mathrm{SA}_{2}$ & $\mathrm{LeA}_{2}$ & $\mathrm{SG}_{2}$ & $\mathrm{LG}_{2}$ \\
\hline $\mathrm{SA}_{3}$ & $\mathrm{LeA}_{3}$ & $\mathrm{SG}_{3}$ & $\mathrm{LG}_{3}$ \\
\hline $\mathrm{SA}_{4}$ & $\mathrm{LeA}_{4}$ & $\mathrm{SG}_{4}$ & $\mathrm{LG}_{4}$ \\
\hline $\mathrm{SA}_{5}$ & $\mathrm{LeA}_{5}$ & $\mathrm{SG}_{5}$ & $\mathrm{LG}_{5}$ \\
\hline $\mathrm{SA}_{6}$ & $\mathrm{LeA}_{6}$ & $\mathrm{SG}_{6}$ & $\mathrm{LG}_{6}$ \\
\hline $\mathrm{SA}_{7}$ & $\mathrm{LeA}_{7}$ & $\mathrm{SG}_{7}$ & $\mathrm{LG}_{7}$ \\
\hline$\cdot$ & $\cdot$ & $\cdot$ & $\cdot$ \\
\hline$\cdot$ & $\cdot$ & $\cdot$ & $\cdot$ \\
\hline$\cdot$ & $\cdot$ & $\cdot$ & $\cdot$ \\
\hline $\mathrm{SA}_{n-1}$ & $\mathrm{LeA}_{n-1}$ & $\mathrm{SG}_{n-1}$ & $\mathrm{LG}_{n-1}$ \\
\hline $\mathrm{SA}_{n}$ & $\mathrm{LeA}_{n}$ & $\mathrm{SG}_{n}$ & $\mathrm{LG}_{n}$ \\
\hline $\mathrm{MSA}_{1}$ & $\mathrm{MLeA}_{1}$ & $\mathrm{MSG}_{1}$ & $\mathrm{MLG}_{1}$ \\
\hline $\mathrm{MSA}_{2}$ & $\mathrm{MLeA}_{2}$ & $\mathrm{MSG}_{2}$ & $\mathrm{MLG}_{2}$ \\
\hline $\mathrm{MSA}_{3}$ & $\mathrm{MLeA}_{3}$ & $\mathrm{MSG}_{3}$ & $\mathrm{MLG}_{3}$ \\
\hline $\mathrm{MSA}_{4}$ & $\mathrm{MLeA}_{4}$ & $\mathrm{MSG}_{4}$ & $\mathrm{MLG}_{4}$ \\
\hline$\ldots$ & $\ldots$ & $\ldots$ & $\ldots$ \\
\hline $\mathrm{MSA}_{L}$ & $\mathrm{MLeA}_{L}$ & $\mathrm{MSG}_{2}$ & $\mathrm{MLG}_{2}$ \\
\hline
\end{tabular}

Figure 8. Set 2: OR + LA1 dataset.

\begin{tabular}{|c|c|c|c|}
\hline $\mathrm{SA}_{5}$ & $\mathrm{LeA}_{5}$ & $\mathrm{SG}_{5}$ & $\mathrm{LG}_{5}$ \\
\hline $\mathrm{SA}_{2}$ & $\mathrm{LeA}_{2}$ & $\mathrm{SG}_{2}$ & $\mathrm{LG}_{2}$ \\
\hline MsA1 & MLeA1 & $\mathrm{MsG}_{1}$ & $\mathrm{MLG}_{1}$ \\
\hline $\mathrm{SA}_{3}$ & $\mathrm{LeA}_{3}$ & $\mathrm{SG}_{3}$ & $\mathrm{LG}_{3}$ \\
\hline $\mathrm{MSA}_{3}$ & MLeA3 & $\mathrm{MsG}_{3}$ & MLG3 \\
\hline $\mathrm{SA}_{n}$ & $\mathrm{LeA}_{n}$ & $\mathrm{SG}_{n}$ & $\mathrm{LG}_{n}$ \\
\hline $\mathrm{MSA}_{4}$ & MLeA4 & $\mathrm{MsG}_{4}$ & $\mathrm{MLG}_{4}$ \\
\hline $\mathrm{SA}_{1}$ & $\mathrm{LeA}_{1}$ & $\mathrm{SG}_{1}$ & $\mathrm{LG}_{1}$ \\
\hline $\mathrm{SA}_{7}$ & $\mathrm{LeA}_{7}$ & $\mathrm{SG}_{7}$ & $\mathrm{LG}_{7}$ \\
\hline $\mathrm{MSAL}$ & MLeAL & MSGL & $\mathrm{MLGL}_{L}$ \\
\hline . & . & . & . \\
\hline . & . & . & . \\
\hline . & . & . & . \\
\hline $\mathrm{MSA}_{2}$ & MLeA2 & $\mathrm{MsG}_{2}$ & $\mathrm{MLG}_{2}$ \\
\hline $\mathrm{SA}_{n-1}$ & $\operatorname{LeA}_{n-1}$ & $\mathrm{SG}_{n-1}$ & $\mathrm{LG}_{n-1}$ \\
\hline
\end{tabular}

Figure 9. Randomly shuffled (OR + LA1 + SH) feature set 2 . 


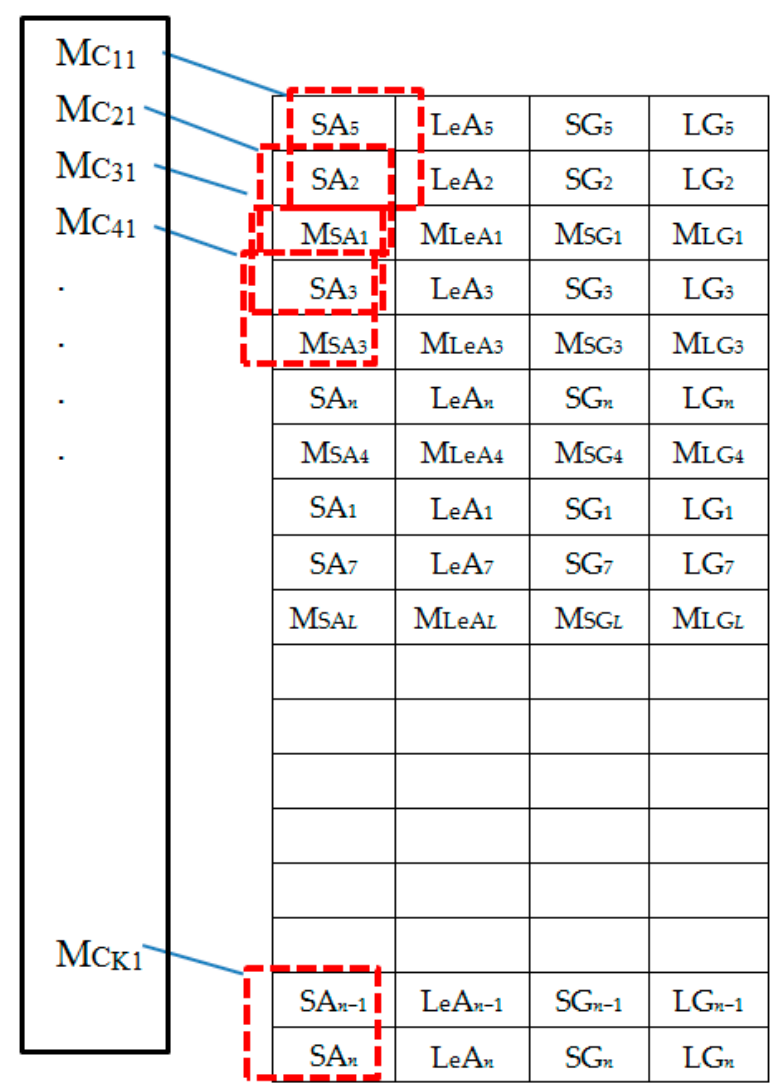

$G=2$ : Local averages

Figure 10. Generating local averages of the shuffled feature set.

\begin{tabular}{|c|c|c|c|}
\hline $\mathrm{SA}_{5}$ & $\mathrm{LeA}_{5}$ & $\mathrm{SG}_{5}$ & LG5 \\
\hline $\mathrm{SA}_{2}$ & $\mathrm{LeA}_{2}$ & $\mathrm{SG}_{2}$ & $\mathrm{LG}_{2}$ \\
\hline $\mathrm{MSA}_{1}$ & MLeA $_{1}$ & $\mathrm{MsG}_{1}$ & MLG $1_{1}$ \\
\hline $\mathrm{SA}_{3}$ & $\mathrm{LeA}_{3}$ & $\mathrm{SG}_{3}$ & $\mathrm{LG}_{3}$ \\
\hline $\mathrm{SA}_{n}$ & LeA $n$ & $\mathrm{SG}_{n}$ & $\mathrm{LG}_{n}$ \\
\hline MsA4 & MLeA4 & $\mathrm{MsG}_{4}$ & $\mathrm{MLG}_{4}$ \\
\hline $\mathrm{SA}_{6}$ & $\mathrm{LeA}_{6}$ & $\mathrm{SG}_{6}$ & $\mathrm{LG}_{6}$ \\
\hline $\mathrm{SA}_{1}$ & $\mathrm{LeA}_{1}$ & $\mathrm{SG}_{1}$ & $\mathrm{LG}_{1}$ \\
\hline $\mathrm{SA}_{7}$ & $\mathrm{LeA}_{7}$ & $\mathrm{SG}_{7}$ & $\mathrm{LG}_{7}$ \\
\hline MSAL & MLeAL & $\mathrm{MsGL}$ & MLGL \\
\hline . & . & . & . \\
\hline . & . & . & . \\
\hline $\mathrm{MSA}_{2}$ & MLeA2 & $\mathrm{MsG}_{2}$ & $\mathrm{MLG}_{2}$ \\
\hline $\mathrm{SA}_{n-1}$ & $\operatorname{LeA}_{n-1}$ & $S G_{n-1}$ & $\mathrm{LG}_{n-1}$ \\
\hline $\mathrm{MC}_{11}$ & $\mathrm{MC}_{12}$ & $\mathrm{MD} 11_{11}$ & $\mathrm{MD} 12_{2}$ \\
\hline $\mathrm{MC}_{21}$ & $\mathrm{MC}_{22}$ & $\mathrm{MD}_{21}$ & $\mathrm{MD}_{22}$ \\
\hline $\mathrm{MC}_{31}$ & $\mathrm{MC}_{32}$ & $\mathrm{MD}_{11}$ & $\mathrm{MD}_{32}$ \\
\hline . & . &. & . \\
\hline $\mathrm{MCK}_{1}$ & MCK2 & MDK1 & MDK2 \\
\hline
\end{tabular}

Figure 11. Set 3: OR + LA1 + SH + LA2 feature set with local averages. 
The results in the variance shifts after each augmentation approach are shown in Figure 12. Figure $12 \mathrm{~b}, \mathrm{c}, \mathrm{e}, \mathrm{f}, \mathrm{h}, \mathrm{i}$ all shows a reduced variance in the augmented data. This is phenomenon is desirable to reduce on overfitting.

In order to further analyse the observations in Figure 12, a description of the feature vector structure is done in this paragraph. We shall use $S$ and $L$ as 50 for illustration. If $Q_{j}, \ldots, Q_{n}$ are the items of a feature vector $u_{i}$, then the feature vector items $Q_{1}, \ldots, Q_{n}$ are structured as follows: (1) $Q_{1}, \ldots, Q_{50}$ are the least 50 spectral densities from the accelerations spectrogram, (2) $Q_{51}, \ldots, Q_{100}$ are the largest 50 spectral densities from the accelerations spectrogram, (3) $Q_{101}, \ldots, Q_{150}$ are the least 50 spectral densities from the gyroscopes spectrogram, (4) $Q_{151}, \ldots, Q_{200}$ are the least 50 spectral densities from the gyroscopes spectrogram.

In Figure 12a-c, it is observed that only the largest part of the gyroscope's spectral information is represented. The implication of this observation is that the huge signal wave forms in a standing scenario is mainly generated by the gyroscope but not the accelerometer. Worth noting is that our standing activity scenario was indeed one without much motion by the arm apart from some few turns.

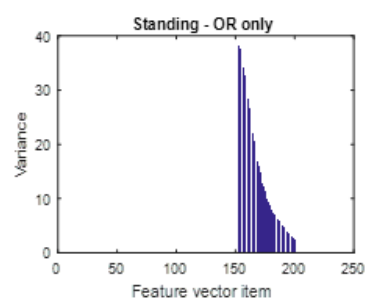

(a)

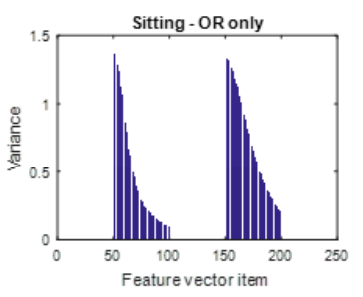

(d)

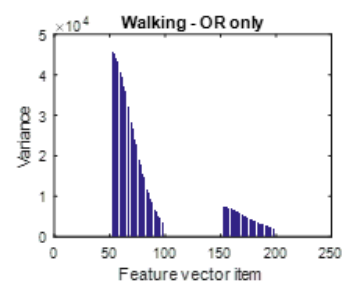

(g)

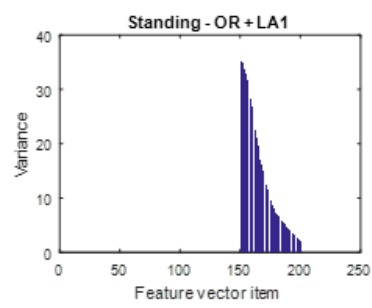

(b)

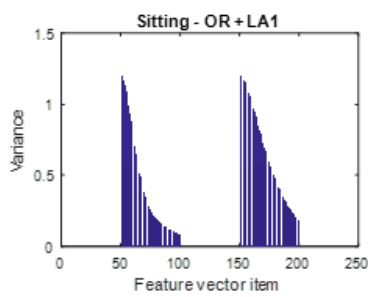

(e)

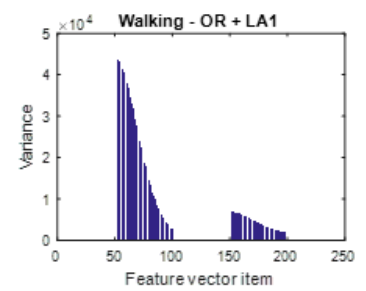

(h)

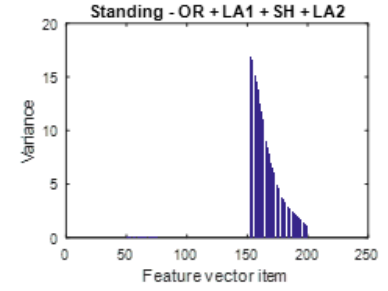

(c)

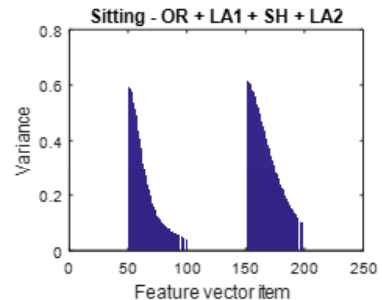

$(\mathbf{f})$

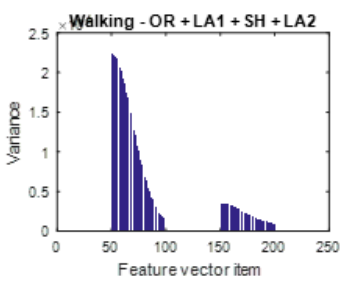

(i)

Figure 12. Visualizing the variance of data to check the influence of each data augmentation block. $(\mathbf{a}, \mathbf{b}, \mathbf{c})$ represent the variance of the unaugmented dataset, augmented dataset after the first local averaging and that of the augmented dataset after the first local averaging, shuffling and the second local averaging procedure respectively for the standing activity. $(\mathbf{d}, \mathbf{e},(\mathbf{f})$ represent the variance of the unaugmented dataset, augmented dataset after the first local averaging and that of the augmented dataset after the first local averaging, shuffling and the second local averaging procedure respectively for the sitting activity. $(\mathrm{g}, \mathbf{h}, \mathbf{i})$ represent the variance of the unaugmented dataset, augmented dataset after the first local averaging and that of the augmented dataset after the first local averaging, shuffling and the second local averaging procedure respectively for the walking activity. 


\section{LSTM Overview and Experimental Setup}

\subsection{LSTM Overview}

In this section, we describe the deep learning model (recurrent neural network (RNN)-LSTM model) that is used for activity recognition in this study. LSTM is a composition from RNN and can as well learn complex temporal dynamics by mapping input sequences to a sequence of hidden states and hidden states to outputs. The niche in LSTM is its ability to learn long term dependencies. In RNN the output responses $h_{i}$ are calculated based on the inputs $x_{i}$ and the responses $h_{i-1}$ from the previous time slot:

$$
\mathbf{h}_{t}=\theta\left(\mathbf{W}_{x h} X_{t}+\mathbf{W}_{h h} h_{t-1}+\mathbf{b}_{h}\right)
$$

where $\theta(\cdot)$ denotes the activation function, $\mathbf{b}_{h}$ is the bias vector, $\mathbf{W}_{x h}$ is the matrix of the weights between the input and hidden layer and $\mathbf{W}_{h h}$ is the matrix of recurrent weights from the hidden layer to itself at adjacent time steps which is used for exploring temporal dependency. An LSTM cell is equipped with an input gate $\mathbf{i}_{t}$, forget gate $\mathbf{f}_{t}$, a cell $c_{t}$ and output response $h_{t}$ all defined as follows based on Figure 13:

$$
\begin{aligned}
& \mathbf{i}_{t}=\sigma\left(\mathbf{W}_{x i} x_{t}+\mathbf{W}_{h i} c_{t-1}+\mathbf{b}_{i}\right) \\
& \mathbf{f}_{t}=\sigma\left(\mathbf{W}_{x f} x_{t}+\mathbf{W}_{h f} h_{t-1}+\mathbf{W}_{c f} c_{t-1}+\mathbf{b} f\right) \\
& \mathbf{c}_{t}=\mathbf{f}_{t} \otimes c_{t-1}+\mathbf{i}_{t} \otimes \tanh \left(\mathbf{W}_{x c} x_{t}+\mathbf{W}_{h c} h_{t-1}+\mathbf{b}_{c}\right) \\
& \mathbf{o}_{t}=\sigma\left(\mathbf{W}_{x o} x_{t}+\mathbf{W}_{h o} h_{t-1}+\mathbf{W}_{c o} c_{t}+\mathbf{b}_{o}\right), \\
& \mathbf{h}_{t}=\mathbf{o}_{t} \otimes \tanh \left(c_{t}\right),
\end{aligned}
$$

where $\otimes$ denotes the elementwise product, $\sigma(x)$ is the sigmoid function defined as $\sigma(x)=1 /\left(1+e^{-x}\right)$, $\mathbf{W}_{\alpha \beta}$ is the weight matrix between $\alpha$ and $\beta$ (e.g., $\mathbf{W}_{x i}$ is the weight matrix from the inputs $\mathbf{x}_{t}$ to the gates $\mathbf{i}_{t}$ ), and $\mathbf{b}_{\beta}$ denotes the bias term of $\beta$ with $\beta \in\{\mathbf{i}, \mathbf{f}, \mathbf{c}, \mathbf{o}\}$. The forget cell serves a major purpose of ensuring and keeping track of long term dependencies without being affected by the vanishing gradient problem exhibited during training in the traditional RNN.

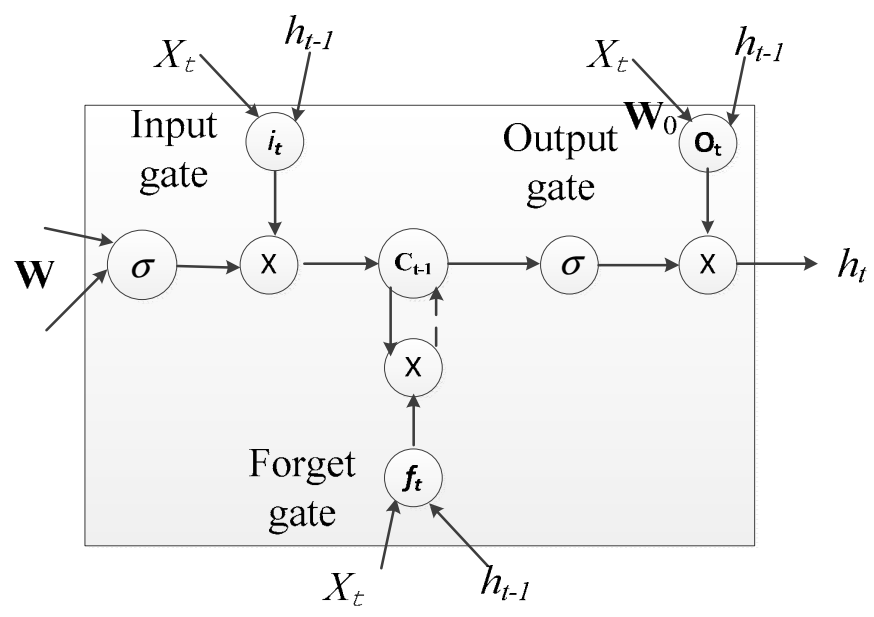

Figure 13. LSTM cell. 
Table 2. Performance metrics.

\begin{tabular}{|c|c|}
\hline Acronym & Description \\
\hline Accuracy & The percentage of correctly predicted samples out of the total number of samples. \\
\hline Precision & $\begin{array}{l}\text { The fraction of the samples which are actually positive among all the samples which } \\
\text { we predicted positive. Precision }=\frac{N_{T P}}{N_{P P}} \text { where, } N_{T P} \text { is the number of true positives and } \\
N_{P P} \text { is the number of predicted positives. }\end{array}$ \\
\hline Recall & $\begin{array}{l}\text { Measures the proportion of positives that are correctly identified. Recall }=\frac{N_{T P}}{N_{A P}} \text { Where, } \\
N_{T P} \text { is the number of true positives and } N_{A P} \text { is the actual number of positives. }\end{array}$ \\
\hline f1_score & The weighted harmonic means of precision and recall. \\
\hline
\end{tabular}

Table 3. Experimental setup.

\begin{tabular}{cc}
\hline Description & Value \\
\hline Number of hidden nodes & 15 \\
\hline Learning rates tested & $0.002,0.006,0.003,0.015,0.01$ \\
\hline Mini batch size & 8000 \\
\hline Loss & 0.001 \\
\hline Regularization & L2 \\
\hline Activation function & RELU (rectified linear unit) \\
\hline Number of training samples (OR + LA1 + SH + LA2) & 9616 \\
\hline Number of training samples (OR + LA1) & 5132 \\
\hline Number of training samples (OR only) & 2614 \\
\hline Number of test samples & Adam optimizer \\
\hline Optimization (back propagation) &
\end{tabular}

The training process for an LSTM network is based on the back-propagation process with the objective of minimizing the error. In the training process, propagation is done towards the last hidden nodes and backwards until the set number of iterations has reached. After this stage, propagation shifts to the SoftMax layer for classification. Each LSTM is stacked with 5 LSTM layers. The neural network performance is evaluated using the performance metrics shown in Table 2. The training parameters for the neural network and their values are stated in Table 3.

The LSTM neural network is comprised of 15 hidden layers. This is considered a small number to take care of the neural network training time. The learning rates are fixed to values between 0 and 1 but closer to 0 because lower learning rates have higher chances of producing higher accuracies. Again, the learning rates are is set between 0 and 1 because the significant values of the RELU activation function exist between 0 and 1 . The mini batch size is fixed because batch processing is used to improve on the training speed. The L2 regularization function is used to control the overfitting of data. The Adam optimizer is used for training the neural network through back propagation. In order to build and test the learning model, training samples and testing samples were collected. A sample in each case is made up of either a 100 -feature vector or a 200 -feature vector.

\subsection{Data Collection Setup}

The accelerometer and gyroscope 3D data were collected from five subjects of ages between 25 and 40 with the IMU sensor tied on their left wrist like a wrist watch. The data transmission rate for the IMU data transmission was set to $10 \mathrm{~Hz}$. The raw data collected is then manipulated through the feature extraction process described in Section 3.1 to form either a 100-feature vector dataset or a 200 -feature vector dataset. These 100 -feature and 200 -feature vector dataset are our dataset in this article. 


\section{Results and Discussion}

To understand the performance of our proposed algorithms, we show in this subsection the influence of the different parts of the algorithms. First, we fix the batch size for our experiments by arbitrarily using four batch sizes (see Table 4) which are all tested on our 200-feature vector dataset and then pick out the batch size that provides the best accuracy while using 0.0002 as the learning rate.

Table 4. Batch size versus accuracy (OR + LA1 + SH + LA2).

\begin{tabular}{cc}
\hline Batch Size & Accuracy \\
\hline 4500 & 76.95 \\
4000 & 80.51 \\
6000 & 82.8 \\
8000 & 88.14 \\
\hline
\end{tabular}

\subsection{Initial Feature Set Performance}

We check the performance of the feature extraction algorithm without any data augmentation by varying the size of the feature vector. We collected our own 3D raw accelerometer and gyroscope data for walking, sitting and standing. Our proposed feature extraction approach described in Section 3.1 is applied to the raw data and generates a 100 and a 200-feature vector dataset which we use for analysis. The two feature sizes have been strategically chosen to represent a small or limited dataset and another with a fairly well represented data set. This serves well in testing how good a feature set is and fit for comparative testing of augmentation performance.

Figure 14 shows that the 200-feature vector dataset performs better than the 100 -feature vector at all learning rates with the best accuracy at $88.7 \%$ and a learning rate of 0.003 . This performance phenomenon is in tandem with the notion that learning with more features should offer a higher degree of accuracy.

Table 5 shows the results from using only OR (without augmentation) with varying learning rates for the 100-feature vector dataset. It should be noted here that the performance of the 100-feature data set is very bad when learning rates of 0.01 and 0.015 are applied. By observing the confusion matrices, it can be said that the dataset is either unbalanced or the size of the dataset is too small for learning to be achieved. It is for such reasons that we intend to show the effect of performing data augmentation on such a dataset. Figure 14 shows the detailed performance of the 100 -feature dataset with various performance indicators.

Table 5. Confusion matrices: (OR only) -100-feature vector datasets.

\begin{tabular}{|c|c|c|c|c|c|c|c|c|c|c|c|c|c|c|c|c|}
\hline \multicolumn{2}{|c|}{$\begin{array}{c}\text { Learning } \\
\text { Rate }\end{array}$} & \multicolumn{3}{|c|}{ (a) 0.0002} & \multicolumn{3}{|c|}{ (b) 0.006} & \multicolumn{3}{|c|}{ (c) 0.003} & \multicolumn{3}{|c|}{ (d) 0.015} & \multicolumn{3}{|c|}{ (e) 0.01} \\
\hline \multirow{3}{*}{$\begin{array}{l}\text { True } \\
\text { label }\end{array}$} & ST & 441 & 240 & 0 & 0 & 681 & 0 & 409 & 272 & 0 & 0 & 0 & 681 & 0 & 0 & 681 \\
\hline & SI & 113 & 560 & 0 & 0 & 673 & 0 & 98 & 575 & 0 & 0 & 0 & 673 & 0 & 0 & 673 \\
\hline & WA & 0 & 1 & 700 & 0 & 2 & 699 & 0 & 1 & 700 & 0 & 0 & 701 & 0 & 0 & 701 \\
\hline & & ST & SI & WA & ST & SI & WA & ST & SI & WA & ST & SI & WA & ST & SI & WA \\
\hline
\end{tabular}




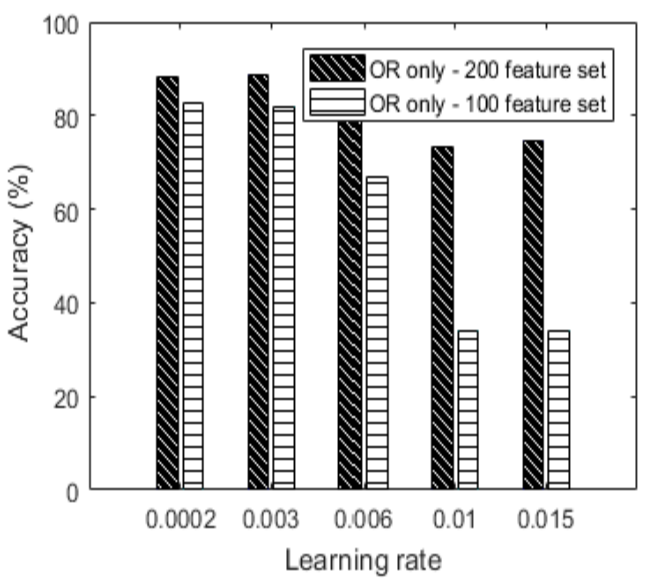

(a)

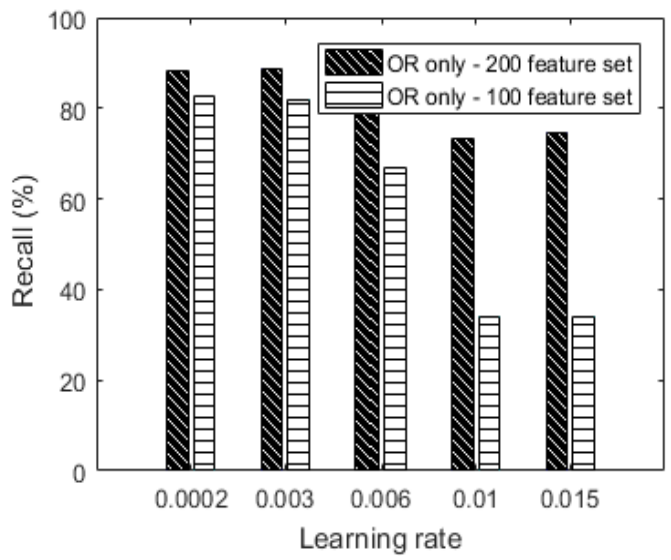

(c)

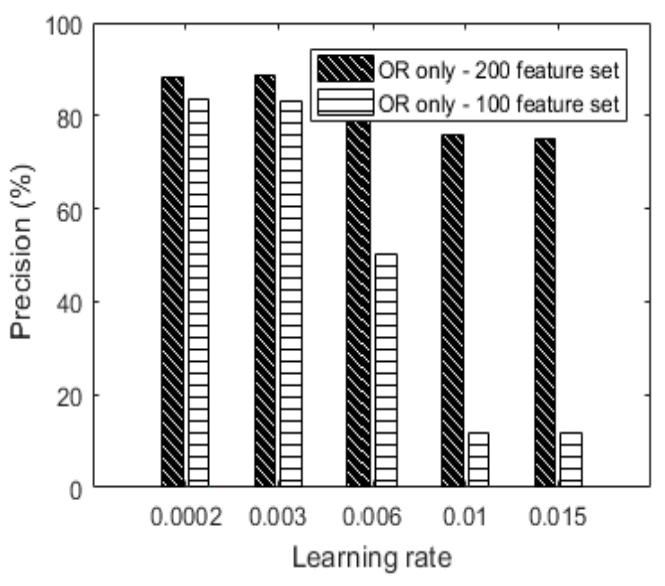

(b)

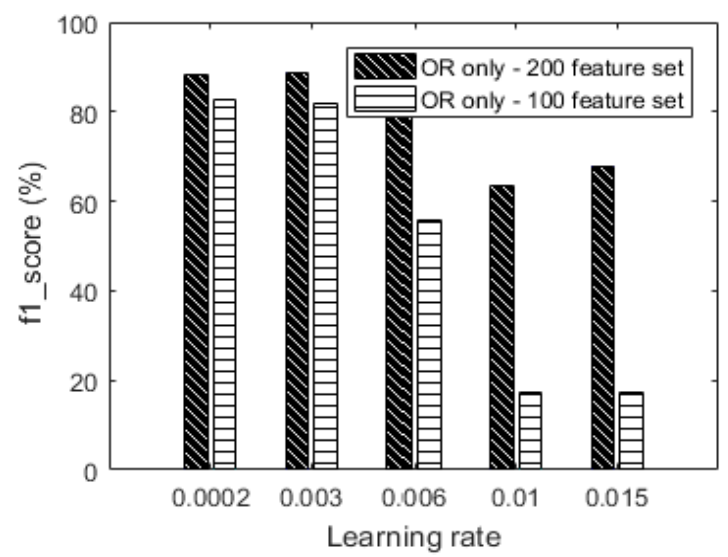

(d)

Figure 14. (a) Accuracy versus learning rate based on only the OR dataset without augmentation, (b) precision versus learning rate based on only the OR dataset without augmentation, (c) recall versus learning rate based on only the OR dataset without augmentation and (d) f1_score versus learning rate based on only the OR dataset without augmentation.

Table 6 shows the results from using only OR (without augmentation) with varying learning rates for the 200-feature vector dataset. By observing the confusion matrices, the 200-feature vector dataset exhibits a more balanced dataset compared to the 100-feature vector dataset. At least all classes are recognizable using all the learning rates. It can be concluded that the greater vector size in a feature set contributed to the accuracy performance seen in Figure 14.

Table 6. Confusion matrices: (OR only)—200-feature vector dataset.

\begin{tabular}{|c|c|c|c|c|c|c|c|c|c|c|c|c|c|c|c|c|}
\hline \multicolumn{2}{|c|}{$\begin{array}{c}\text { Learning } \\
\text { Rate }\end{array}$} & \multicolumn{3}{|c|}{ (a) 0.0002} & \multicolumn{3}{|c|}{ (b) 0.006} & \multicolumn{3}{|c|}{ (c) 0.003} & \multicolumn{3}{|c|}{ (d) 0.015} & \multicolumn{3}{|c|}{ (e) 0.01} \\
\hline \multirow{3}{*}{$\begin{array}{l}\text { True } \\
\text { label }\end{array}$} & ST & 547 & 153 & 0 & 550 & 149 & 1 & 555 & 145 & 0 & 81 & 580 & 39 & 13 & 656 & 31 \\
\hline & SI & 154 & 1066 & 0 & 168 & 1052 & 0 & 150 & 1070 & 0 & 33 & 1177 & 10 & 3 & 1207 & 10 \\
\hline & WA & 0 & 0 & 694 & 1 & 0 & 693 & 0 & 0 & 694 & 0 & 0 & 694 & 1 & 0 & 693 \\
\hline & & ST & SI & WA & ST & SI & WA & ST & SI & WA & ST & SI & WA & ST & SI & WA \\
\hline
\end{tabular}


The 100 spectral features dataset exhibits a lesser training time of about $1 \mathrm{~h}$ and 45 min compared to about $2 \mathrm{~h}$ and $30 \mathrm{~min}$ for the larger 200 feature dataset.

\subsection{Data Augmentation Performance}

Each feature vector size is analyzed independently to understand the performance of each augmentation block. We start with the 100-feature vector dataset. Table 7 shows the recognition results of the dataset augmented by the local average (where $G=4$ ). Compared to the confusion matrices earlier represented in Table 5 of the same dataset, it is evident that Table 7 presents more balanced dataset especially for Table $7 \mathrm{~b}, \mathrm{~d}$,e with reference to Table $5 \mathrm{~b}, \mathrm{~d}, \mathrm{e}$.

Next, we show the influence of data augmentation by OR $+\mathrm{LA} 1+\mathrm{SH}+\mathrm{LA} 2$. Table 8 shows the confusion matrices and Figure 15 includes the summarized results for OR + LA1 + SH + LA2 It should be noted that the LA2 after shuffling was done with $G$ specified as $G=2$. The results of this augmentation show that lower learning rates results into approximately similar recognition accuracies, however for higher learning rates, of $0.006,0.01$ and 0.015 the accuracy is significantly low compared to both the OR and OR + LA1 results. From this observation, it can be concluded that the parameter $G$ in our augmentation algorithm is critical in determining augmentation performance by local averaging.

Summarily, by comparing the accuracy results in Figure 16 to the OR only results in the same Figure 16, the OR + LA1 augmentation achieves significant accuracy improvement by $32.75 \%$ for 0.01 learning rate, $32.7 \%$ for 0.015 learning rate, and $3.89 \%$ for 0.006 learning rate.

The augmentation performance of the 200-feature vector dataset is now considered. Preliminary results (Table 6 and Figure 14) without augmentation show that the data imbalance problem isn't significant in the dataset. Tables 9 and 10 show the confusion matrices for the dataset augmented by only local averaging (where $G=4$ in LA1 and $G=2$ in LA2). The results in Figure 16 show that our augmentations provide almost similar results for low learning rates at 0.0002 and 0.003 . However, at higher learning rates OR + LA1 competes both OR and OR + LA1 + SH + LA2. Table 10 shows the confusion matrices for the OR + LA1 + SH + LA2-200-feature vector dataset.

By comparing OR and OR + LA1 + SH + LA2 results in Figure 16 respectively, it should be noted that at a learning rate of 0.01 , a significant improvement of up to $4.24 \%$ in accuracy is observed. This observation is also true for the 100-feature vector dataset exhibiting $32.75 \%$ improvement in accuracy.

Summarily, the effect of our augmentation approach is seen to be more effective in the lesser feature vector size dataset. The approach is seen to help improve on lower recognition accuracy caused by the data imbalance problem.

Table 7. Confusion matrices: (OR + LA1)-100-feature vector dataset.

\begin{tabular}{|c|c|c|c|c|c|c|c|c|c|c|c|c|c|c|c|c|}
\hline \multicolumn{2}{|c|}{$\begin{array}{c}\text { Learning } \\
\text { Rate }\end{array}$} & \multicolumn{3}{|c|}{ (a) 0.0002} & \multicolumn{3}{|c|}{ (b) 0.006} & \multicolumn{3}{|c|}{ (c) 0.003} & \multicolumn{3}{|c|}{ (d) 0.015} & \multicolumn{3}{|c|}{ (e) 0.01} \\
\hline \multirow{3}{*}{$\begin{array}{l}\text { True } \\
\text { label }\end{array}$} & ST & 437 & 244 & 0 & 97 & 584 & 0 & 416 & 265 & 0 & 0 & 681 & 0 & 0 & 680 & 1 \\
\hline & SI & 106 & 567 & 0 & 18 & 655 & 0 & 103 & 570 & 0 & 0 & 673 & 0 & 0 & 673 & 0 \\
\hline & WA & 0 & 1 & 700 & 1 & 0 & 700 & 0 & 1 & 700 & 0 & 1 & 700 & 0 & 0 & 701 \\
\hline & & ST & SI & WA & ST & SI & WA & ST & SI & WA & ST & SI & WA & ST & SI & WA \\
\hline & & \multicolumn{15}{|c|}{ Pred } \\
\hline
\end{tabular}

Table 8. Confusion matrices: (OR + LA1 + SH + LA2) -100-feature vector dataset.

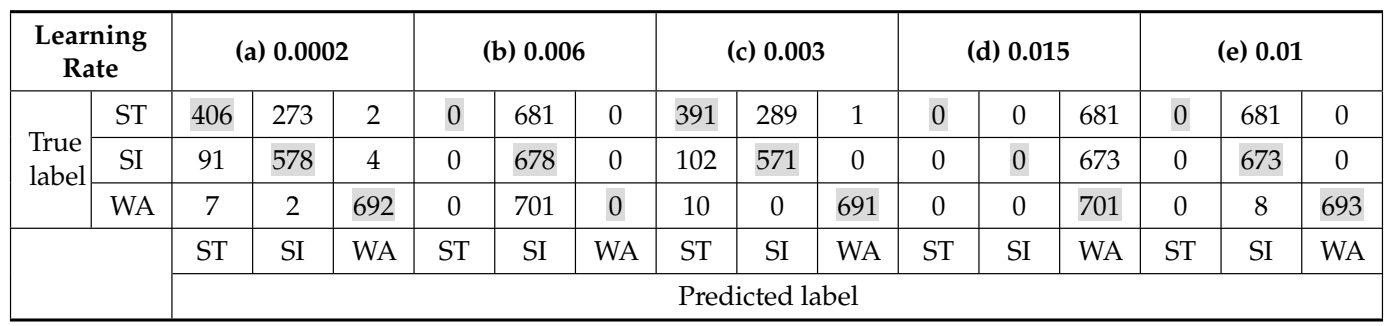




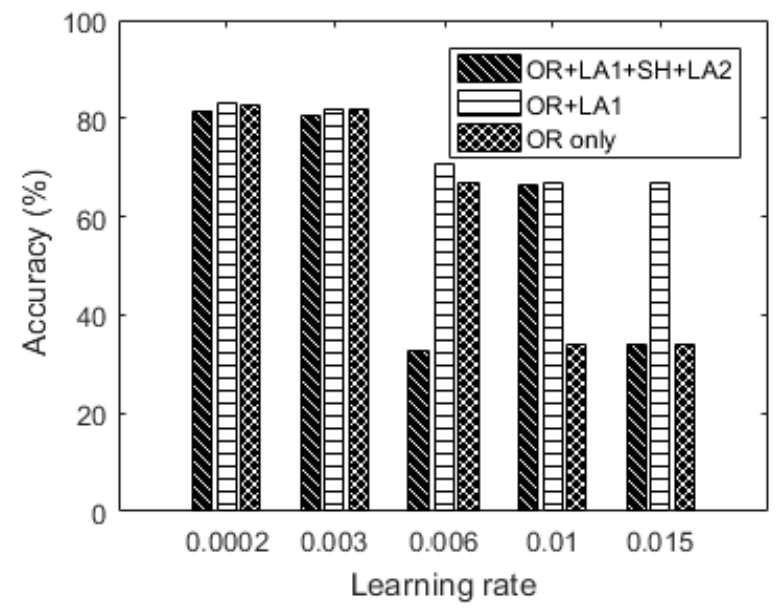

(a)

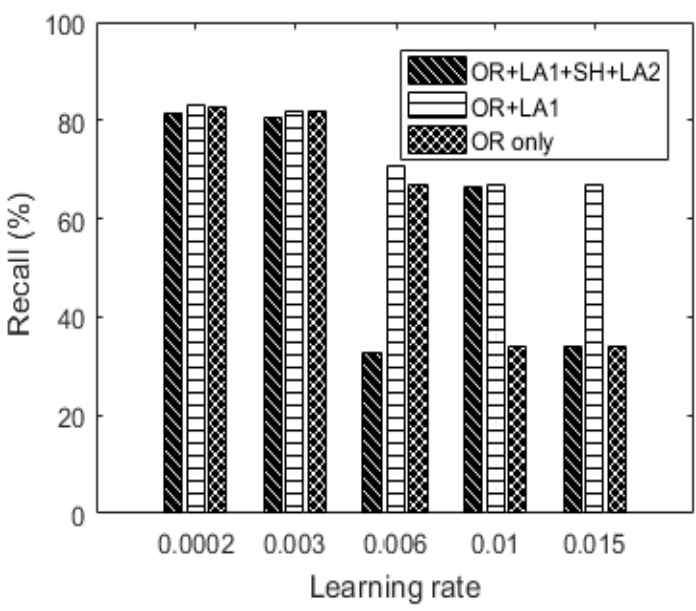

(c)

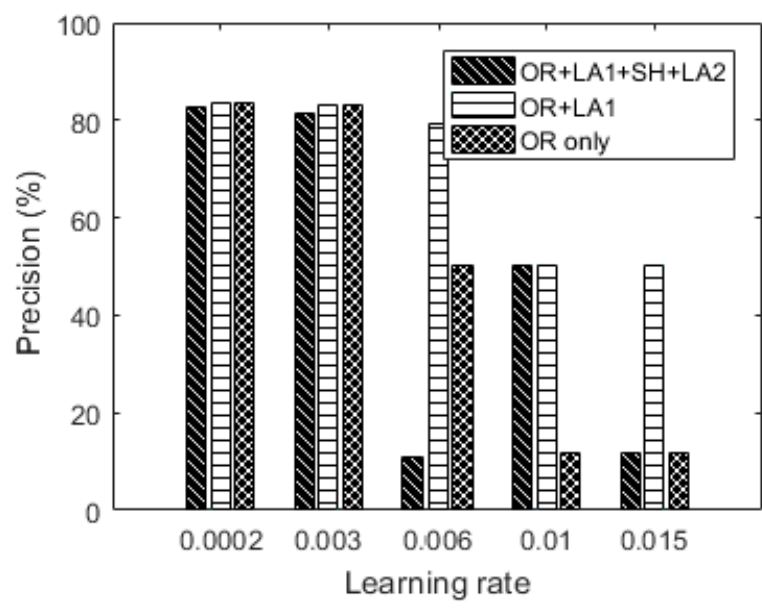

(b)

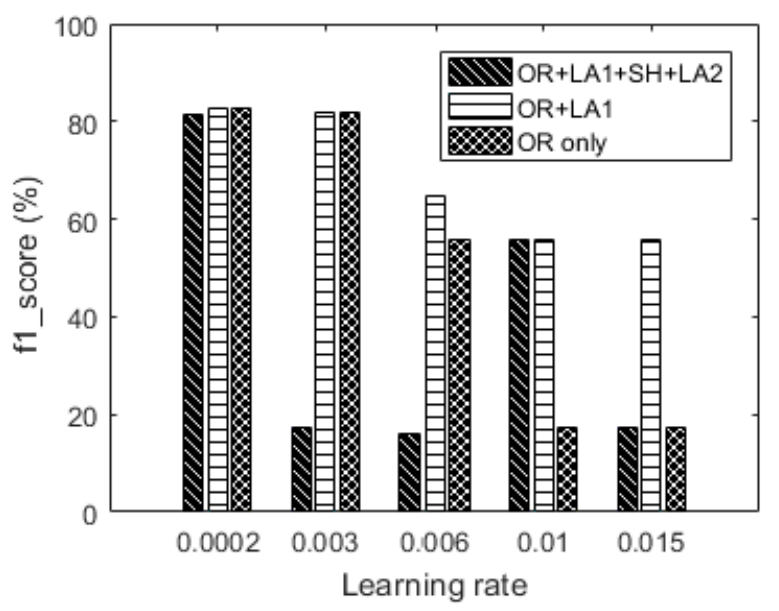

(d)

Figure 15. 100-feature vector dataset: (a) Accuracy versus learning rate, (b) precision versus learning rate, (c) recall versus learning rate and (d) f1_score versus learning rate.

Table 9. Confusion matrices: (OR + LA1) -200-feature vector dataset.

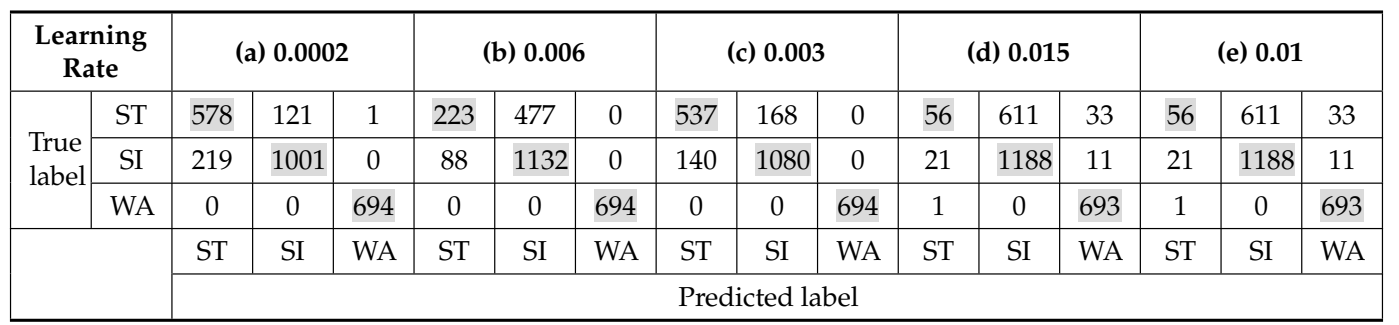

Table 10. Confusion matrices: (OR + LA1 + SH + LA2) -200-feature vector dataset.

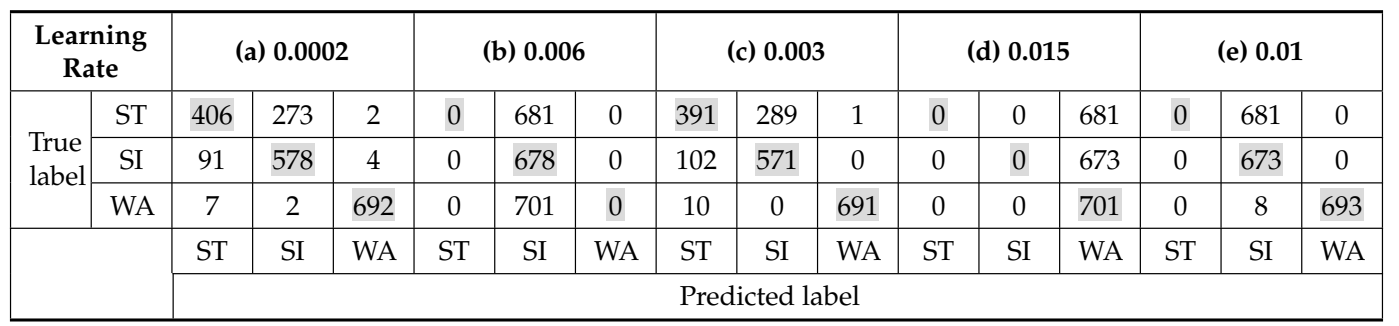




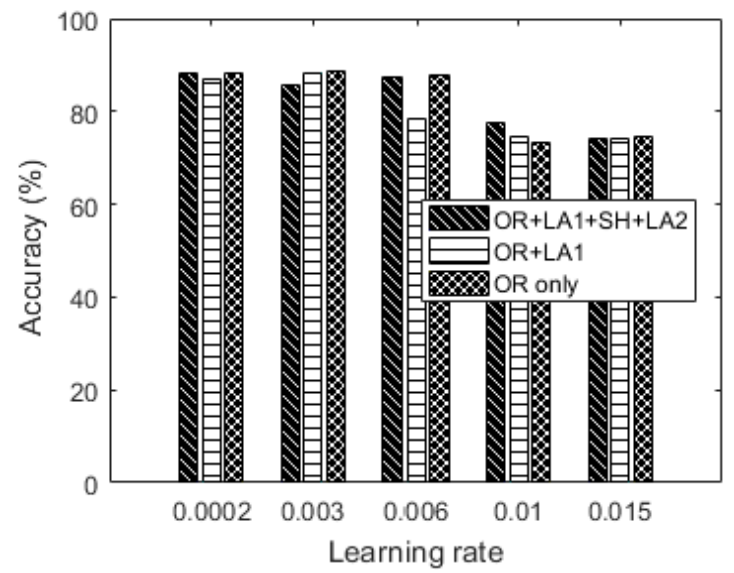

(a)

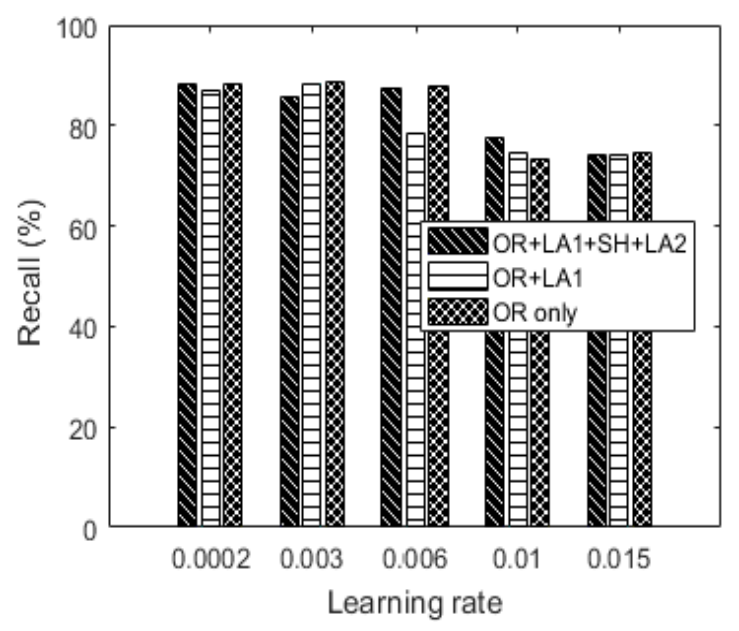

(c)

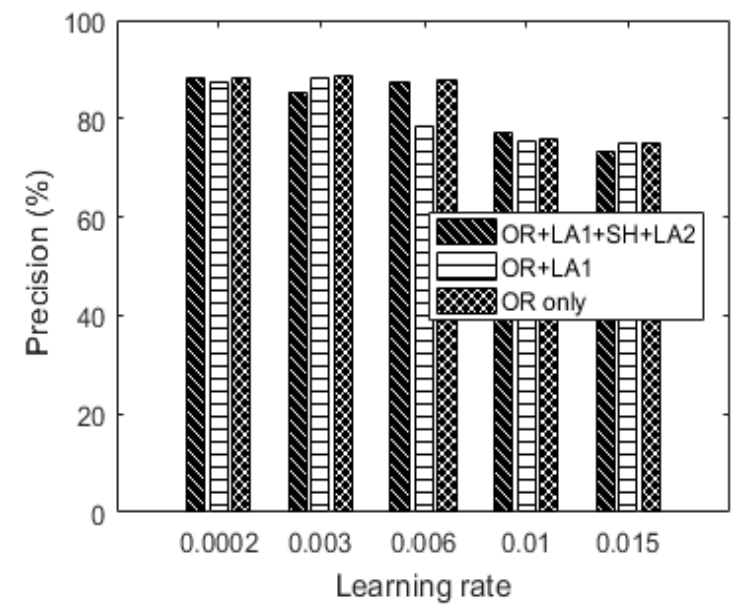

(b)

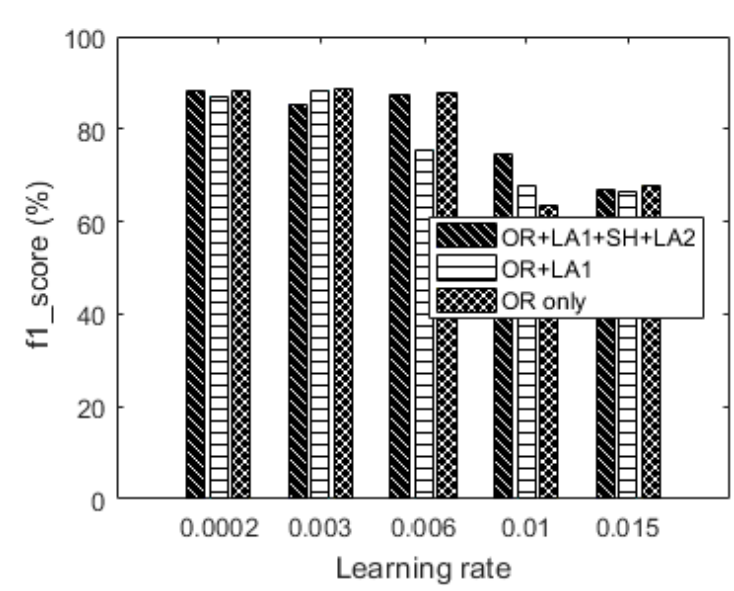

(d)

Figure 16. 200-feature vector dataset: (a) Accuracy versus learning rate, (b) precision versus learning rate, (c) recall versus learning rate and (d) f1_score versus learning rate.

\subsection{Validation of Proposed Algorithm}

In this section, we implement the proposed data augmentation approaches exclusively on a HAR dataset available from the University of California (UCI) machine learning repository [10] to check on its performance. The specific data used in this validation is an extract of the three activities (standing, sitting and walking) from the original dataset. Our intention is to try as much as possible to compare the quality of our dataset with a state-of-the-art dataset based on the proposed feature extractions and augmentations. Again, selecting only three specific activities from the original dataset is fit for pinpointing the unbalanced data problem which is common in discriminating classes ranging from 2 to 3. It is therefore expected that an unbalanced class scenario shall manifest in the experiments hence the need for solutions such as data augmentations. Therefore 3886 training series and 1519 test series were extracted and trained using the same LSTM network settings that are used for training our own datasets. The UCI dataset is a 128-feature vector dataset. The training and test data for the accelerometer and gyroscope were used.

Preliminary augmentation results from our own dataset, showed that OR + LA1 is better than $\mathrm{OR}+\mathrm{LA} 1+\mathrm{SH}+\mathrm{LA} 2$. We have therefore chosen to test OR and OR + LA1 for our validation. Note that: (1) OR for the UCI dataset represents the original online dataset without any manipulation by the authors of this paper. (2) The OR + LA1 for the UCI dataset represents the original UCI online dataset 
which the authors in this paper have manipulated by performing the first local averaging procedure refered to as LA1 in this paper and then used as augmentation data. Tables 11 and 12 show the OR+ LA1 and OR only confusion matrix results. The graphical results on accuracy, precision, recall and f1_score are shown in Figures 17 and 18. Both OR and OR + LA1 performance results are shown in Figures 17 and 18 for comparison.

Table 11. Confusion matrices: (OR + LA1).

\begin{tabular}{|c|c|c|c|c|c|c|c|c|c|c|c|c|c|c|c|c|}
\hline \multicolumn{2}{|c|}{$\begin{array}{c}\text { Learning } \\
\text { Rate }\end{array}$} & \multicolumn{3}{|c|}{ (a) 0.0002} & \multicolumn{3}{|c|}{ (b) 0.006} & \multicolumn{3}{|c|}{ (c) 0.003} & \multicolumn{3}{|c|}{ (d) 0.015} & \multicolumn{3}{|c|}{ (e) 0.01} \\
\hline \multirow{3}{*}{$\begin{array}{l}\text { True } \\
\text { label }\end{array}$} & ST & 455 & 67 & 10 & 525 & 0 & 7 & 471 & 57 & 4 & 532 & 0 & 0 & 532 & 0 & 0 \\
\hline & SI & 153 & 337 & 1 & 485 & 1 & 5 & 107 & 383 & 1 & 491 & 0 & 0 & 491 & 0 & 0 \\
\hline & WA & 0 & 0 & 496 & 0 & 0 & 496 & 0 & 0 & 496 & 496 & 0 & 0 & 496 & 0 & 0 \\
\hline & & ST & SI & WA & ST & SI & WA & ST & SI & WA & ST & SI & WA & ST & SI & WA \\
\hline & & \multicolumn{15}{|c|}{ Predicted label } \\
\hline
\end{tabular}

Based on Figure 17, the best accuracy result from the UCI HAR dataset (OR-without augmentation) is $86.7 \%$. With augmentation, the best recognition accuracy is $88.87 \%$. Although this improvement of $1.27 \%$ on accuracy is small, the proposed approach gives a significant improvement of $24.92 \%$ on accuracy using a learning rate of 0.003 . The same learning rate of 0.003 generates the best accuracy and is based on the OR + LA1 augmentation. It can be observed in Table $12 \mathrm{~b}, \mathrm{~d}$, e that the classification is skewed towards some class or classes. The same phenomenon can be seen in Table 11b,d,e.

Table 12. Confusion matrices: (OR only).

\begin{tabular}{|c|c|c|c|c|c|c|c|c|c|c|c|c|c|c|c|c|}
\hline \multicolumn{2}{|c|}{$\begin{array}{c}\text { Learning } \\
\text { Rate }\end{array}$} & \multicolumn{3}{|c|}{ (a) 0.0002} & \multicolumn{3}{|c|}{ (b) 0.006} & \multicolumn{3}{|c|}{ (c) 0.003} & \multicolumn{3}{|c|}{ (d) 0.015} & \multicolumn{3}{|c|}{ (e) 0.01} \\
\hline \multirow{3}{*}{$\begin{array}{l}\text { True } \\
\text { label }\end{array}$} & ST & 480 & 48 & 4 & 532 & 0 & 0 & 319 & 210 & 3 & 0 & 528 & 4 & 0 & 530 & 2 \\
\hline & SI & 145 & 344 & 2 & 491 & 0 & 0 & 325 & 160 & 6 & 0 & 486 & 5 & 0 & 486 & 5 \\
\hline & WA & 3 & 0 & 493 & 496 & 0 & 0 & 0 & 4 & 492 & 0 & 0 & 496 & 0 & 2 & 494 \\
\hline & & ST & SI & WA & ST & SI & WA & ST & SI & WA & ST & SI & WA & ST & SI & WA \\
\hline & & \multicolumn{15}{|c|}{ Predicted label } \\
\hline
\end{tabular}

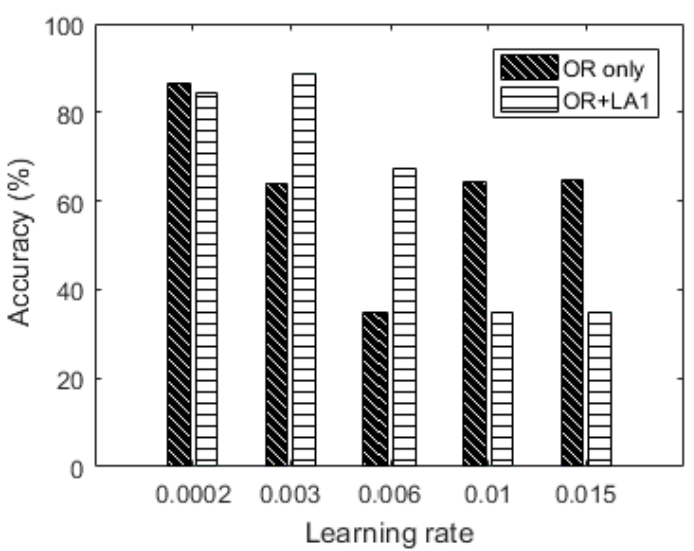

(a)

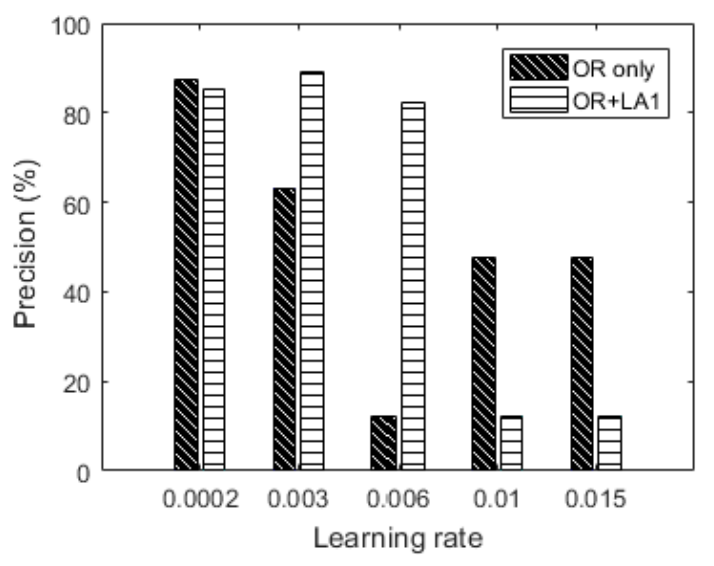

(b)

Figure 17. Cont. 


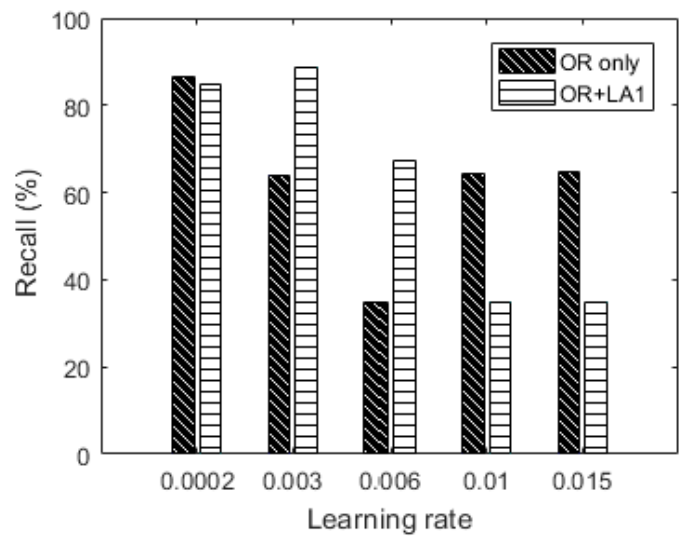

(c)

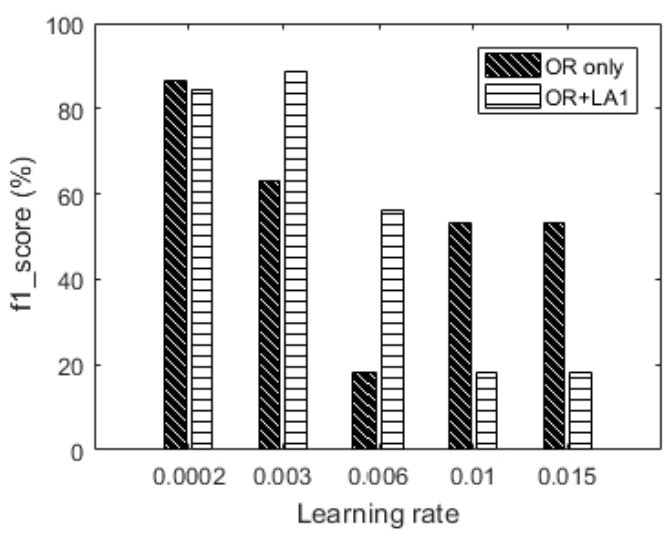

(d)

Figure 17. UCI's 128-feature vector dataset: (a) Accuracy versus learning rate, (b) precision versus learning rate, $(\mathbf{c})$ recall versus learning rate and (d) f1_score versus learning rate.

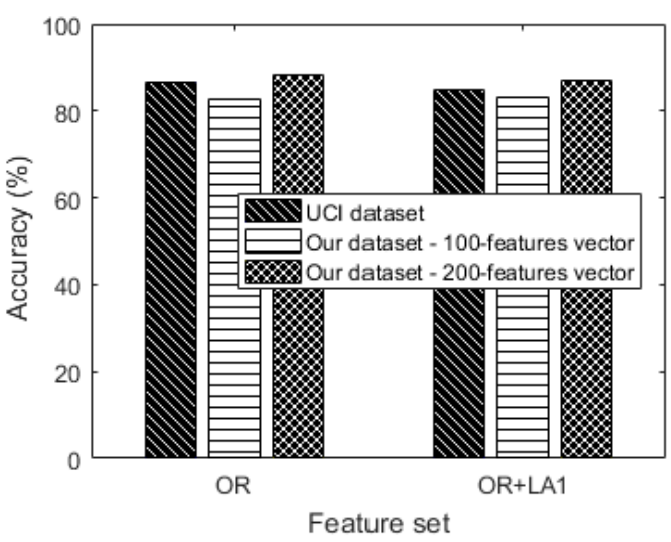

(a)

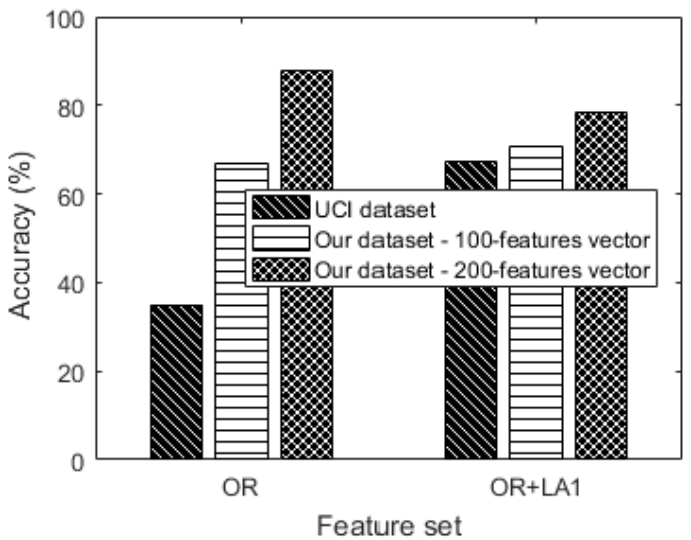

(c)

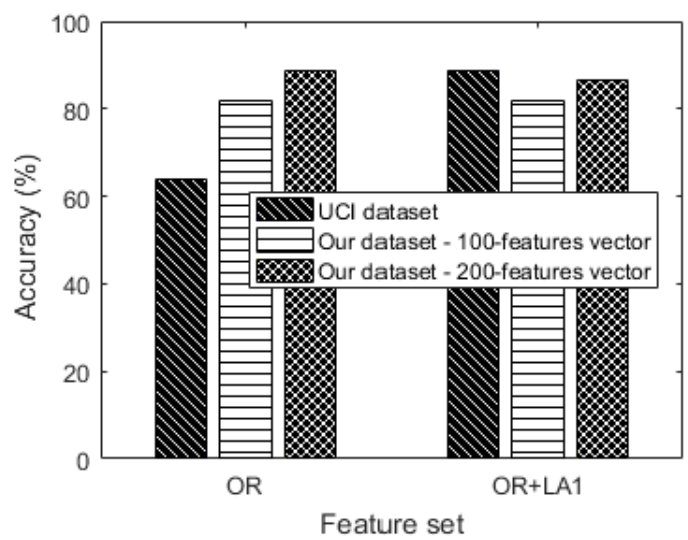

(b)

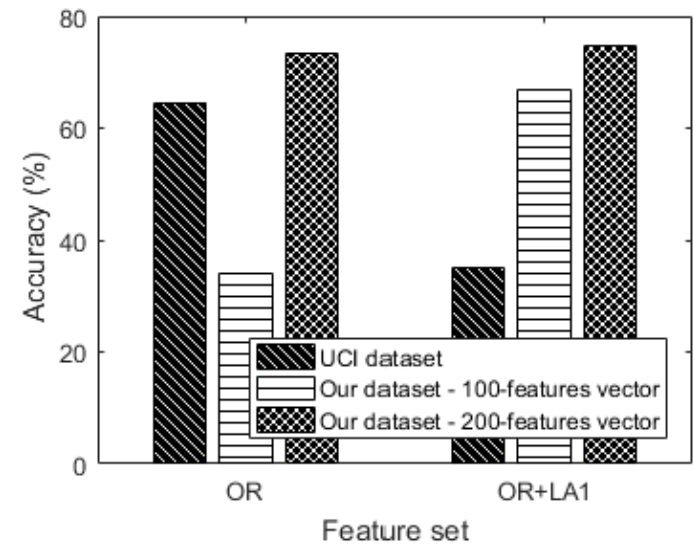

(d)

Figure 18. Comparing the performance of OR and OR + LA1 using the UCI dataset and our dataset at various learning rates of: (a) 0.0002 (b) 0.003 (c) 0.006 and (d) 0.01 .

Figure 18 shows that $\mathrm{OR}+\mathrm{LA} 1$ augmentation is competitive on accuracy at low learning rates when compared to using only OR for classification. Figure 18 shows the results of accuracy for both the 
UCI dataset and our dataset. In Figure 18a, our 200-feature vector dataset outperforms the UCI dataset in both OR and OR + LA1. This could be a result of the greater size of the feature vector. In Figure 18b, Our dataset without any augmentation outperforms the original UCI dataset for both the 100 and 200 -feature vectors dataset by $18 \%$ and $24.81 \%$ in accuracy respectively. OR + LA1 in Figure $18 \mathrm{~b}$ for the UCI dataset shows an improvement in accuracy compared to its OR accuracy result. This shows that OR + LA1 augmentation has had a positive effect on improving the accuracy by $24.95 \%$ for the UCI dataset.

In Figure 18c, our dataset without any augmentation outperforms the UCI dataset for both the 100 and 200 -feature vectors by $31.74 \%$ and $52.77 \%$ respectively. In the OR + LA1 scenario, our dataset out performs the UCI dataset for both the 100 and 200 -feature vector dataset by $3.37 \%$ and $11.1 \%$ respectively. We can also observe in Figure 18c that the OR + LA1 augmentation caused an improvement in the UCI dataset by $32.6 \%$ in accuracy.

In Figure 18d, the performance based on OR is poor for our 100-feature vector dataset compared to both the UCI dataset and the 200-feature vector dataset. This is likely to be an effect of the larger learning rate applied in a small size dataset. In OR + LA1 of Figure 18d, the 200-feature vector dataset relatively maintained its accuracy level while the UCI dataset declined in accuracy. It is also observed that the 100-feature vector dataset improved in accuracy in the OR + LA1 scenario. Generally, Figure 18d especially for the UCI dataset provides insights that, higher learning rates may require careful feature representation especially when data augmentation is a must.

\section{Conclusions and Future Work}

In this paper, we proposed using only spectral features for learning human activity. We showed that using few spectral features we can achieve state-of-the-art recognition performance. Fewer spectral features exhibit a lesser training time of about $1 \mathrm{~h}$ and 45 min compared to about $2 \mathrm{~h}$ and 30 min for the large feature set used in this article. Furthermore, an augmentation ensemble used in feature space was also defined for purposes of improving recognition accuracy. Through greedy tuning of the learning rate, our proposed feature extraction and augmentation ensemble achieved improved recognition accuracy at several learning rates and in a few cases, performs close to the unaugmented feature set. The proposed extraction approach provided the best performance improvement in accuracy of $52.77 \%$ in comparison with the UCI online dataset. The proposed OR + LA1 provided the best performance improvement in accuracy of $32.6 \%$ compared with the UCI online dataset. The LSTM deep learning model was utilized in the study. Therefore, further studies in this work shall be done to compare the performance of the proposed approach on other HAR datasets and various machine learning models.

Author Contributions: Writing-original draft, O.S.E.; Writing-review \& editing, D.S.H.

Funding: This work was supported by Institute for Information \& communications Technology Promotion (IITP) grant funded by the Korea government (MSIT, R7124-16-0004, Development of Intelligent Interaction Technology Based on Context Awareness and Human Intention Understanding).

Conflicts of Interest: The authors declare no conflict of interest.

\section{References}

1. Debes, C.; Merentitis, A.; Sukhanov, S.; Niessen, M.; Frangiadakis, N.; Bauer, A. Monitoring activities of daily living in smart homes: Understanding human behavior. IEEE Signal Process. Mag. 2016, 33, 81-94. [CrossRef]

2. Reiss, A.; Stricker, D. Introducing a new benchmarked dataset for activity monitoring. In Proceedings of the 2012 16th International Symposium on Wearable Computers (ISWC), Newcastle, UK, 18-22 June 2012; pp. 108-109.

3. Sukkarieh, S.; Nebot, E.M.; Durrant-Whyte, H.F. A high integrity IMU/GPS navigation loop for autonomous land vehicle applications. IEEE Trans. Robot. Autom. 1999, 15, 572-578. [CrossRef]

4. Jiménez, A.R.; Seco, F.; Prieto, J.C.; Guevara, J. Indoor pedestrian navigation using an INS/EKF framework for yaw drift reduction and a foot-mounted IMU. In Proceedings of the 20107 th Workshop on Positioning Navigation and Communication (WPNC), Dresden, Germany, 11-12 March 2010; pp. 135-143. 
5. Ojeda, L.; Borenstein, J. Non-GPS navigation for security personnel and first responders. J. Navig. 2007, 60, 391-407. [CrossRef]

6. Xu, J.Y.; Chang, H.-I.; Chien, C.; Kaiser, W.J.; Pottie, G.J. Context-driven, prescription-based personal activity classification: Methodology, architecture, and end-to-end implementation. IEEE J. Biomed. Health Inform. 2014, 18, 1015-1025. [CrossRef] [PubMed]

7. Ravì, D.; Wong, C.; Lo, B.; Yang, G.-Z. A deep learning approach to on-node sensor data analytics for mobile or wearable devices. IEEE J. Biomed. Health Inform. 2017, 21, 56-64. [CrossRef] [PubMed]

8. Ronao, C.A.; Cho, S.-B. Human activity recognition with smartphone sensors using deep learning neural networks. Expert Syst. Appl. 2016, 59, 235-244. [CrossRef]

9. Eyobu, O.S.; Kim, Y.; Han, D.S. Activity Recognition for Infant Health Care Based on Wireless Inertial Measurement Unit Motion Data. In Proceedings of the Korean Institute of Communication Sciences Conference, Jeju, Korea, 21-23 June 2017; p. 436.

10. Anguita, D.; Ghio, A.; Oneto, L.; Parra, X.; Reyes-Ortiz, J.L. A Public Domain Dataset for Human Activity Recognition using Smartphones. In Proceedings of the European Symposium on Artificial Neural Networks, Computational Intelligence and Machine Learning, Bruges, Belgium, 24-26 April 2013.

11. Yang, J.; Zhang, D.; Frangi, A.F.; Yang, J.-Y. Two-dimensional PCA: A new approach to appearance-based face representation and recognition. IEEE Trans. Pattern Anal. Mach. Intell. 2004, 26, 131-137. [CrossRef] [PubMed]

12. Ebied, H.M. Feature extraction using PCA and Kernel-PCA for face recognition. In Proceedings of the 2012 8th International Conference on Informatics and Systems (INFOS), Cairo, Egypt, 14-16 May 2012; p. MM-72-MM-77.

13. Ohashi, H.; Al-Nasser, M.; Ahmed, S.; Akiyama, T.; Sato, T.; Nguyen, P.; Nakamura, K.; Dengel, A. Augmenting Wearable Sensor Data with Physical Constraint for DNN-Based Human-Action Recognition. Presented at ICML 2017 Times Series Workshop, Sydney, Australia, 6-11 August 2017.

14. Um, T.T.; Pfister, F.M.J.; Pichler, D.; Endo, S.; Lang, M.; Hirche, S.; Fietzek, U.; Kulić, D. Data Augmentation of Wearable Sensor Data for Parkinson's Disease Monitoring using Convolutional Neural Networks. In Proceedings of the 19th ACM International Conference on Multimodal Interaction, Glasgow, UK, 13-17 November 2017.

15. Salamon, J.; Bello, J.P. Deep convolutional neural networks and data augmentation for environmental sound classification. IEEE Signal Process. Lett. 2017, 24, 279-283. [CrossRef]

16. Sun, Y.; Kamel, M.S.; Wong, A.K.; Wang, Y. Cost-sensitive boosting for classification of imbalanced data. Pattern Recognit. 2007, 40, 3358-3378. [CrossRef]

17. He, H.; Garcia, E.A. Learning from imbalanced data. IEEE Trans. Knowl. Data Eng. 2008, 21, 1263-1284.

18. Jeni, L.A.; Cohn, J.F.; de la Torre, F. Facing Imbalanced Data-Recommendations for the Use of Performance Metrics. In Proceedings of the 2013 Humaine Association Conference on Affective Computing and Intelligent Interaction (ACII), Geneva, Switzerland, 2-5 September 2013; pp. 245-251.

19. van Hulse, J.; Khoshgoftaar, T.M.; Napolitano, A. Experimental perspectives on learning from imbalanced data. In Proceedings of the 24th International Conference on Machine Learning, Corvallis, OR, USA, 20-24 June 2007; pp. 935-942.

20. Saito, T.; Rehmsmeier, D.M. The precision-recall plot is more informative than the ROC plot when evaluating binary classifiers on imbalanced datasets. PLoS ONE 2015, 10, e0118432. [CrossRef] [PubMed]

21. Kwolek, B.; Kepski, M. Human fall detection on embedded platform using depth maps and wireless accelerometer. Comput. Methods Prog. Biomed. 2014, 117, 489-501. [CrossRef] [PubMed]

22. Mao, A.; Ma, X.; He, Y.; Luo, J. Highly portable, sensor-based system for human fall monitoring. Sensors 2017, 17, 2096. [CrossRef] [PubMed]

23. Pierleoni, P.; Belli, A.; Palma, L.; Pellegrini, M.; Pernini, L.; Valenti, S. A high reliability wearable device for elderly fall detection. IEEE Sensors J. 2015, 15, 4544-4553. [CrossRef]

24. Dai, H.; Zhang, P.; Lueth, T.C. Quantitative assessment of parkinsonian tremor based on an inertial measurement unit. Sensors 2015, 15, 25055-25071. [CrossRef] [PubMed]

25. Yu, Z.; Lee, M. Human motion based intent recognition using a deep dynamic neural model. Robot. Auton. Syst. 2015, 71, 134-149. [CrossRef]

26. Chen, C.; Jafari, R.; Kehtarnavaz, N. A survey of depth and inertial sensor fusion for human action recognition. Multimed. Tools Appl. 2017, 76, 4405-4425. [CrossRef] 
27. Laptev, I. On space-time interest points. Int. J. Comput. Vis. 2005, 64, 107-123. [CrossRef]

28. Bobick, A.F.; Davis, J.W. The recognition of human movement using temporal templates. IEEE Trans. Pattern Anal. Mach. Intell. 2001, 23, 257-267. [CrossRef]

29. Ding, M.; Fan, G. Multilayer Joint Gait-Pose Manifolds for Human Gait Motion Modeling. IEEE Trans. Cybern. 2015, 45, 2413-2424. [CrossRef] [PubMed]

30. Tunca, C.; Pehlivan, N.; Ak, N.; Arnrich, B.; Salur, G.; Ersoy, C. Inertial sensor-based robust gait analysis in non-hospital settings for neurological disorders. Sensors 2017, 17, 825. [CrossRef] [PubMed]

31. Bruno, B.; Mastrogiovanni, F.; Sgorbissa, A. Wearable inertial sensors: Applications, challenges, and public test benches. IEEE Robot. Autom. Mag. 2015, 22, 116-124. [CrossRef]

32. Bert, F.; Giacometti, M.; Gualano, M.R.; Siliquini, R. Smartphones and health promotion: A review of the evidence. J. Med. Syst. 2014, 38, 9995. [CrossRef] [PubMed]

33. Bächlin, M.; Förster, K.; Tröster, G. SwimMaster: A wearable assistant for swimmer. In Proceedings of the 11th International Conference on Ubiquitous Computing, Orlando, FL, USA, 30 September-3 October 2009; pp. 215-224.

34. Avci, A.; Bosch, S.; Marin-Perianu, M.; Marin-Perianu, R.; Havinga, P. Activity recognition using inertial sensing for healthcare, wellbeing and sports applications: A survey. In Proceedings of the 2010 23rd international conference on Architecture of Computing Systems (ARCS), Hannover, Germany, 22-25 February 2010; pp. 1-10.

35. Ravi, N.; Dandekar, N.; Mysore, P.; Littman, M.L. Activity recognition from accelerometer data. In Proceedings of the 17th conference on Innovative Applications of Artificial Intelligence, Pittsburgh, PA, USA, 9-13 July 2005; pp. 1541-1546.

36. Pirttikangas, S.; Fujinami, K.; Nakajima, T. Feature selection and activity recognition from wearable sensors. In Proceedings of the International Symposium on Ubiquitious Computing Systems, Seoul, Korea, 11-13 October 2006; pp. 516-527.

37. Kwapisz, J.R.; Weiss, G.M.; Moore, S.A. Activity recognition using cell phone accelerometers. ACM SIGKDD Explor. Newsl. 2011, 12, 74-82. [CrossRef]

38. Yang, J.-Y.; Wang, J.-S.; Chen, Y.-P. Using acceleration measurements for activity recognition: An effective learning algorithm for constructing neural classifiers. Pattern Recog. Lett. 2008, 29, 2213-2220. [CrossRef]

39. Eyben, F.; Wöllmer, M.; Schuller, B. Opensmile: The munich versatile and fast open-source audio feature extractor. In Proceedings of the 18th ACM International Conference on Multimedia, Firenze, Italy, 25-29 October 2010; pp. 1459-1462.

40. Maurer, U.; Smailagic, A.; Siewiorek, D.P.; Deisher, M. Activity recognition and monitoring using multiple sensors on different body positions. In Proceedings of the International Workshop on Wearable and Implantable Body Sensor Networks, Cambridge, MA, USA, 3-5 April 2006; pp. 4-116.

41. Ward, J.A.; Lukowicz, P.; Tröster, G. Gesture spotting using wrist worn microphone and 3-axis accelerometer. In Proceedings of the 2005 Joint Conference on Smart Objects and Ambient Intelligence: Innovative Context-Aware Services: Usages and Technologies, Grenoble, France, 12-14 October 2005; pp. 99-104.

42. Bulling, A.; Ward, J.A.; Gellersen, H.; Troster, G. Eye movement analysis for activity recognition using electrooculography. IEEE Trans. Pattern Anal. Mach. Intell. 2011, 33, 741-753. [CrossRef] [PubMed]

43. Huynh, T.; Schiele, B. Analyzing features for activity recognition. In Proceedings of the 2005 Joint Conference on Smart Objects and Ambient Intelligence: Innovative Context-Aware Services: Usages and Technologies, Grenoble, France, 12-14 October 2005; pp. 159-163.

44. Wang, N.; Ambikairajah, E.; Lovell, N.H.; Celler, B.G. Accelerometry based classification of walking patterns using time-frequency analysis. In Proceedings of the 29th Annual International Conference of the IEEE Engineering in Medicine and Biology Society, Lyon, France, 22-26 August 2007; pp. 4899-4902.

45. le Guennec, A.; Malinowski, S.; Tavenard, R. Data Augmentation for Time Series Classification using Convolutional Neural Networks. In Proceedings of the ECML/PKDD Workshop on Advanced Analytics and Learning on Temporal Data, Porto, Portugal, 11 September 2016.

46. Cui, Z.; Chen, W.; Chen, Y. Multi-scale convolutional neural networks for time series classification. arXiv 2016, arXiv:1603.06995.

47. Wong, S.C.; Gatt, A.; Stamatescu, V.; McDonnell, M.D. Understanding data augmentation for classification: When to warp? In Proceedings of the 2016 International Conference on Digital Image Computing: Techniques and Applications (DICTA), Gold Coast, Australia, 30 November-2 December 2016; pp. 1-6. 
48. DeVries, T.; Taylor, G.W. Dataset Augmentation in Feature Space. arXiv 2017, arXiv:1702.05538.

49. Chawla, N.V.; Bowyer, K.W.; Hall, L.O.; Kegelmeyer, W.P. SMOTE: Synthetic minority over-sampling technique. J. Artif. Intell. Res. 2002, 16, 321-357. [CrossRef]

50. Guo, X.; Singh, S.; Lee, H.; Lewis, R.L.; Wang, X. Deep learning for real-time Atari game play using offline Monte-Carlo tree search planning. In Proceedings of the 27th International Conference on Neural Information Processing Systems, Montreal, QC, Canada, 8-13 December 2014; pp. 3338-3346. 